\title{
bASES21 - Um Modelo para a Autoavaliação de Habilidades do Século XXI no Contexto do Ensino de Computação na Educação Básica
}

\author{
bASES21 - A Model for the Auto-Assessment of 21th Century Skills in the Context of \\ Teaching Computing in Basic Education
}

\author{
Fernanda Mioto \\ Departamento de Informática e \\ Estatística \\ Universidade Federal de Santa \\ Catarina \\ mioto.f@gmail.com
}

\author{
Giani Petri \\ Universidade Federal de Santa \\ Maria \\ Programa de Pós-Graduação em \\ Ciência da Computação \\ Universidade Federal de Santa \\ Catarina \\ gpetri@inf.ufsm.br
}

Christiane Gresse von Wangenheim Departamento de Informática e Estatística

Universidade Federal de Santa Catarina

c.wangenheim@ufsc.br

\author{
Adriano F. Borgatto \\ Departamento de Informática e Estatística \\ Universidade Federal de Santa Catarina \\ adriano.borgatto@ufsc.br
}

\author{
Lúcia H. M. Pacheco \\ Departamento de Informática e Estatística \\ Universidade Federal de Santa Catarina \\ lucia.pacheco@ufsc.br
}

\begin{abstract}
Resumo
Na sociedade do conhecimento, na qual vivemos atualmente, saber interpretar, buscar, comunicar e compartilhar novas informações são habilidades chaves para um cidadão bem-sucedido. Estas habilidades, conhecidas como habilidades do século XXI, incluem pensamento crítico, trabalho em equipe, comunicação, entre outras. Atualmente, existem diversas propostas de como integrar o aprendizado dessas habilidades na educação básica, inclusive por meio do ensino da computação. No entanto, partindo da hipótese de que o ensino da computação pode contribuir para o aprendizado de habilidades do século XXI, existem poucas evidencias sistematicamente coletadas para confirmar esta afirmação. Uma razão para essa ausência é a carência de modelos de avaliação de habilidades do século XXI. Assim, o objetivo deste trabalho é desenvolver e avaliar um modelo para a avaliação das habilidades do século XXI no contexto do ensino da computação na educação básica. Com base nos resultados de um mapeamento da literatura, o modelo bASES21 é sistematicamente desenvolvido assim como o seu instrumento de medição, um questionário de autoavaliação. Uma avaliação inicial em termos de confiabilidade e validade do instrumento de medição, com base em 148 respostas de estudantes da educação básica, indica uma alta confiabilidade interna (alfa de Cronbach $=0$,958). Os resultados, quanto a validade do instrumento, foram inconclusivos. Mesmo algumas habilidades demonstrando correlação interna, identificou-se como oportunidade de melhoria uma possível reclassificação dos itens com base em uma análise mais detalhada com uma amostra maior. Assim, o modelo bASES21 representa um primeiro passo suportando a avaliação de habilidades do século XXI, visando a melhoria sistemática do ensino da computação na educação básica.
\end{abstract}

Palavras-Chave: Habilidades do Século XXI; Modelo; Avaliação; Ensino de Computação; Educação Básica.

Cite as: Mioto, F., Petri, G., Gresse Von Wangenheim, C., Borgatto, A. F., Pacheco, L. H. M. (2019). bASES21 - A Model for the Auto-Assessment of 21th Century Skills in the Context of Teaching Computing in Basic Education (bASES21 - Um Modelo para a Autoavaliação de Habilidades do Século XXI no Contexto do Ensino de Computação na Educação Básica). Brazilian Journal of Computers in Education (Revista Brasileira de Informática na Educação - RBIE), 27(1), 26-57. DOI: 10.5753/RBIE.2019.27.01.26 


\begin{abstract}
In today's knowledge society, knowing how to interpret, search, communicate and share new information are key skills for a successful citizen. Such skills, known as $21^{\text {st }}$ century skills, include critical thinking, teamwork, communication, among others. Currently, there are several proposals on how to integrate the learning of these skills in basic education, including through the teaching of computing. Yet, based on the hypothesis that teaching computing can contribute to the learning of 21 st century skills, there is few systematically evidence available to support this claim. One reason for this absence is the lack of 21 st century skills assessment models. Thus, the objective of this article is to develop and evaluate a model for the assessment of 21st century skills in the context of teaching computing in basic education. Based on the results of a mapping study, the bASES21 model is systematically developed as well as its measurement instrument, a self-assessment questionnaire. An initial assessment in terms of reliability and validity of the measurement instrument, based on 148 responses from students in basic education, indicate a high internal reliability (Cronbach's alpha $=0.958)$. Results regarding the validity of the instrument were inconclusive. Even although some skills demonstrate internal correlation, an improvement opportunity is the possible re-classification of items based on a more detailed analysis with a larger sample. Thus, the bASES21 model represents a first step supporting the assessment of 21 st century skills, aiming at the systematic improvement of teaching computing in basic education.
\end{abstract}

Keywords: $21^{\text {st }}$ Century Skills; Model; Assessment; Computing Education; K-12.

\title{
1 Introdução
}

A maneira como trabalhamos e nos relacionamos, enquanto sociedade, tem mudado severamente nas últimas décadas (Binkley et al., 2011). Um fator propulsor destas mudanças é o rápido desenvolvimento das tecnologias de informação e comunicação (TICs) (Voogt \& Roblin, 2012). Nesta sociedade, caracterizada como a sociedade do conhecimento (Anderson, 2008; Dede, 2010), saber interpretar, buscar, comunicar e compartilhar novas informações são habilidades chaves para o cidadão de hoje em dia. Estas habilidades, que manipulam informações de alguma maneira, são consideradas habilidades do século XXI (P21, 2015).

Existe uma grande discussão sobre a classificação e quais habilidades seriam de fato habilidades do século XXI. Em geral, entende-se que essas habilidades incluem: "Pensamento crítico", "Resolução de problemas", "Trabalho em equipe" e "Criatividade" (AMA, 2012; P21, 2015). Podem ser incluídas também habilidades como "Proficiência em mídia", "Proficiência em TIC (Tecnologia da informação e comunicação)" e "Comunicação" (P21, 2015), além de habilidades referentes à flexibilidade e adaptação, iniciativa e autodidatismo, habilidades sociais, produtividade e liderança (P21, 2015).

Existem muitas maneiras de integrar o ensino de habilidades do século XXI na educação básica e uma delas é por meio do ensino da computação (CSTA, 2017). Segundo a Association for Computing Machinery (ACM, 2005), computação é qualquer atividade voltada à um objetivo que necessite, se beneficie de/ou crie computadores. Dessa maneira, computação é o estudo de computadores e algoritmos, incluindo seus princípios, hardware, design de software, aplicações e o seu impacto na sociedade (CSTA, 2017). O ensino de computação visa desenvolver habilidades como pensamento computacional, colaboração, sistemas de comunicação e seu impacto global e ético na comunidade (CSTA, 2017). Estas habilidades estão relacionadas à definição de algumas das habilidades do século XXI, como pensamento crítico, trabalho em equipe e responsabilidade social.

Além de contribuir para o desenvolvimento de habilidades do século XXI, atualmente, a computação também é uma habilidade fundamental para o mercado de trabalho, tornando essencial a integração do seu ensino na educação básica (EC, 2017). A Sociedade Brasileira de Computação (SBC) defende que o ensino da computação seja integrado à Base Nacional Comum Curricular (SBC, 2017). Segundo a SBC, é fundamental que o Brasil siga os passos de diversos países e inclua em seu currículo o ensino da computação, sendo que o conhecimento 
básico de computação tornou-se hoje tão necessário quanto conhecimento de matemática, física, história e outras ciências (SBC, 2017). No exterior, já existem várias iniciativas com esse propósito, tais como a Code.org ${ }^{1}$, Code.club $^{2}$ e, no Brasil, a iniciativa Computação na Escola ${ }^{3}$, Meninas Digitais ${ }^{4}$, entre outros. Essas iniciativas focam tanto no ensino do pensamento computacional, inclusive por meio de atividades sem computadores, p.ex. o CSUnplugged ${ }^{5}$, como também na prática de programação por meio de atividades usando linguagens visuais de blocos como Scratch ${ }^{6}$, Blockly ${ }^{7}$, Snap! ${ }^{8}$ ou App Inventor ${ }^{9}$.

A atual prática educacional também enfatiza a importância do uso da tecnologia na educação. A tecnologia não é usada apenas para transferência de conhecimento, mas também oportuniza a interconectividade dos alunos em ambientes colaborativos, enriquecendo processos de aprendizagem de construtivismo social, conectivismo e aprendizagem experiencial (Kivunja, 2014). Desta forma, contribui para a aprendizagem ativa, pensamento crítico, resolução de problemas, criatividade e trabalho em equipe no século XXI (Masethe et al., 2017).

Mesmo, observando a importância do ensino da computação para o desenvolvimento de habilidades do século XXI, existem poucas evidências confirmando sua contribuição na prática. A maioria das avaliações realizadas em relação ao impacto da aprendizagem de habilidades do século XXI em atividades realizadas para o ensino de computação na educação básica é voltada à análise da aprendizagem de competências específicas da área, como programação e/ou de pensamento computacional (Grover \& Pea, 2013; Morelli et al., 2010), não avaliando o desenvolvimento das habilidades do século XXI. Por outro lado, existem estudos sobre aprendizagem de habilidades do século XXI de uma forma mais generalizada (Griffin \& Care, 2014), não necessariamente voltados para o contexto de ensino de computação na educação básica. Desta forma, identifica-se uma ausência de avaliações de habilidades do século XXI (Autores, 2018) inseridas no contexto do ensino da computação, em atividades realizadas na educação básica, para assim, obter evidências do seu impacto no desenvolvimento destas habilidades. Uma das razões para esta ausência de avaliações é a falta de modelos de avaliação de habilidades do século XXI.

Assim, o objetivo deste artigo é apresentar o desenvolvimento e avaliação do modelo bASES21 (Assessing $21^{\text {st }}$ CEntury Skills), para a avaliação de habilidades do século XXI no contexto de ensino de computação na educação básica. A partir do modelo desenvolvido, propõe-se um instrumento de autoavaliação que possa ser usado para medir o impacto do ensino da computação no aprendizado de habilidades do século XXI em atividades conduzidas para alunos da educação básica. Os resultados dessa pesquisa representam um passo inicial voltado ao fornecimento de um suporte para a avaliação do impacto de atividades realizadas para ensinar computação na educação básica para o desenvolvimento de habilidades do século XXI. E assim, contribuir na seleção e criação de atividades eficazes para o desenvolvimento das habilidades, contribuindo na formação de cidadãos.

\footnotetext{
${ }^{1}$ code.org

${ }^{2}$ www.codeclubbrasil.org.br

${ }^{3}$ www.computacaonaescola.ufsc.br

${ }^{4}$ meninas.sbc.org.br/

${ }^{5}$ csunplugged.org

${ }^{6}$ scratch.mit.edu

${ }^{7}$ blockly-games.appspot.com/

${ }^{8}$ snap.berkeley.edu

${ }^{9}$ appinventor.mit.edu
} 


\section{Fundamentação Teórica}

\subsection{Ensino da Computação na Educação Básica}

Segundo o K-12 Computer Science Framework (2016) a introdução do ensino da computação na educação básica se tornou importante pois é necessário que as crianças/jovens aprendam não somente a usar o computador, mas também a criar artefatos novos utilizando um computador.

No Brasil, a educação básica é composta pela educação infantil, ensino fundamental e ensino médio (BNCC, 2018). A educação infantil é focada para crianças de até 5 anos de idade e tem como objetivo ensinar as crianças a conviver, brincar, participar entre outras atividades. Já o ensino fundamental é dividido entre os anos iniciais, sendo esses do $1^{\circ}$ ao $5^{\circ}$ ano, e os anos finais, do $6^{\circ}$ ao $9^{\circ}$ ano. O ensino médio compõe os anos finais da educação básica e é dividido em três séries. Tanto o ensino fundamental quanto o ensino médio têm foco em quatro áreas do conhecimento: línguas, matemática, ciências humanas e ciências da natureza (BNCC, 2018). Atualmente, a Base Nacional Comum Curricular não inclui o ensino da computação em nenhuma das áreas de conhecimento, que é considerado fundamental para educação básica segundo a Sociedade Brasileira de Computação (SBC, 2017).

O ensino da computação na educação básica prevê o desenvolvimento de estratégias para resolver problemas (SBC, 2017). O ensino da computação também pode ser visto como proporcionador de uma nova ferramenta de comunicação, utilizando o computador para expressar e criar ideias novas, dando imenso poder ao seu usuário (CSTA, 2017). O K-12 Computer Science Framework (2016) foi criado visando elaborar um framework para o ensino da computação na educação básica de escopo internacional, sendo elaborado de forma sistemática pela comunidade internacional. O framework identifica os principais conceitos e as principais práticas que devem ser abordadas no ensino da computação na educação básica (Figura 1).
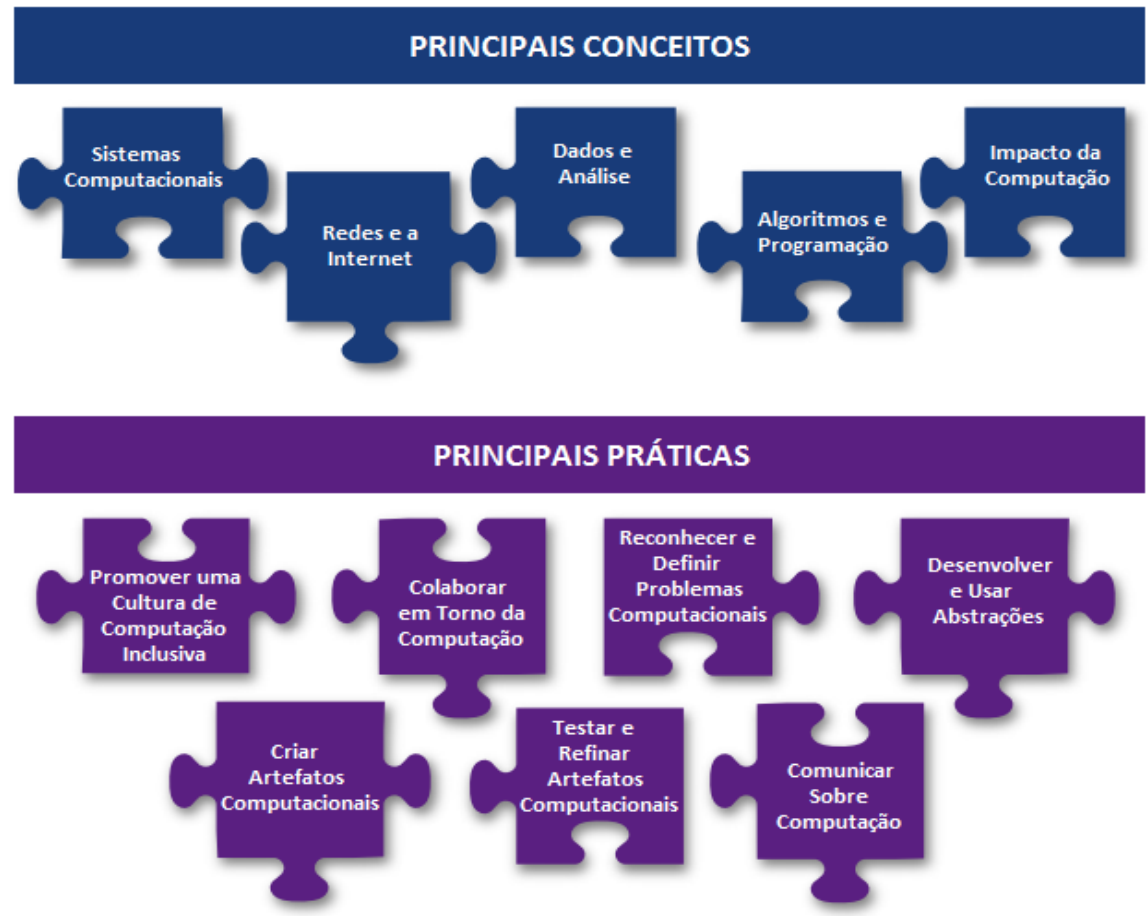

Figura 1: Principais conceitos e práticas (Traduzido de K-12 CSF, 2016). 
As principais práticas não só abordam os conceitos de computação, definidos anteriormente, mas também proporcionam o desenvolvimento de habilidades do século XXI. Na Tabela 1 é apresentada a comparação das práticas propostas pelo K-12 Computer Science Framework (2016) com habilidades do século XXI.

Tabela 1: Habilidades do século XXI vs. práticas de computação (traduzido de P21, 2017).

\begin{tabular}{|l|l|}
\hline $\begin{array}{l}\text { Habilidades do } \\
\text { século XXI }\end{array}$ & Principais práticas \\
\hline Criatividade & $\begin{array}{l}\text { Cria artefatos computacionais com o objetivo de praticidade, expressão pessoal ou para } \\
\text { abordar uma questão social. }\end{array}$ \\
\hline Inovação & Modificar um artefato existente para melhorá-lo ou customizá-lo. \\
\hline Pensamento crítico & Avaliar se a solução computacional de um problema é apropriada e factível. \\
\hline $\begin{array}{l}\text { Resolução de } \\
\text { problemas }\end{array}$ & $\begin{array}{l}\text { Decompor um problema real complexo em subproblemas gerenciáveis que possam ser } \\
\text { resolvidos com soluções existentes. }\end{array}$ \\
\hline Comunicação & Descrever, justificar e documentar processos e soluções computacionais. \\
\hline Colaboração & Receber e oferecer feedback construtivo a outros membros da equipe. \\
\hline
\end{tabular}

Ao comparar as práticas com as habilidades do século XXI é possível perceber como o ensino da computação proporciona um ambiente natural para o desenvolvimento de habilidades do século XXI. Desta maneira é notável a contribuição do ensino da computação para o desenvolvimento de habilidades do século XXI (P21, 2017).

\subsection{Habilidades do Século XXI}

Habilidades do século XXI são habilidades que os estudantes precisam desenvolver para que consigam prosperar em uma sociedade altamente tecnológica e volátil. Muitas dessas novas habilidades são referentes ao aperfeiçoamento de habilidades como pensamento analítico, resolução de problemas e colaboração, sendo essas habilidades diferentes das habilidades acadêmicas tradicionais, baseadas em conhecimento conceituais (Dede, 2010).

Existem diversas definições das habilidades do século XXI criado por diferentes iniciativas, incluindo ATC21 (Binkley et al., 2011), UNESCO (Delors et al., 1996), OECD (OECD, 2005) e o P21 (P21, 2015), como apresentado na Tabela 2. Observa-se, em geral, que essas principais referências de definição de habilidades do século XXI abordam os mesmos assuntos indicados pelas categorias. Somente o modelo da OECD não aborda habilidades referentes a categoria de aprender e inovação. Com exceção do modelo da UNESCO, os modelos refinam essas categorias em habilidades, variando somente em termos de agrupamento, nomenclatura e de grau de detalhamento. 
Tabela 2: Comparativo de definições de habilidades do século XXI.

\begin{tabular}{|c|c|c|c|c|}
\hline \multicolumn{5}{|c|}{ Modelos de definições de habilidades do século XXI } \\
\hline Modelo & $\begin{array}{c}\text { ATC21 } \\
\text { (Binkley et al., 2011) }\end{array}$ & $\begin{array}{c}\text { UNESCO (Delors et } \\
\text { al., 1996) }\end{array}$ & $\begin{array}{c}\text { OECD } \\
(\text { OECD, 2005) }\end{array}$ & $\begin{array}{c}\text { P21 } \\
\text { (P21, 2015) }\end{array}$ \\
\hline Categoria & Formas de pensar & Aprendendo a saber & -- & Aprender e inovação \\
\hline Habilidades & $\begin{array}{l}\text { Criatividade e } \\
\text { inovação; } \\
\text { Pensamento crítico, } \\
\text { solução de problemas e } \\
\text { tomada de decisão; } \\
\text { Aprender a aprender e } \\
\text { metacognição }\end{array}$ & -- & -- & $\begin{array}{l}\text { Criatividade; } \\
\text { Pensamento crítico; } \\
\text { Resolução de } \\
\text { problemas; } \\
\text { Comunicação; } \\
\text { Colaboração }\end{array}$ \\
\hline \multirow[t]{2}{*}{ Categoria } & Formas de trabalhar & Aprendendo a fazer & $\begin{array}{l}\text { Interagindo com } \\
\text { grupos heterogêneos }\end{array}$ & Vida e carreira \\
\hline & $\begin{array}{l}\text { Colaboração e trabalho } \\
\text { em equipe; } \\
\text { Comunicação }\end{array}$ & -- & $\begin{array}{l}\text { Se relacionar bem com } \\
\text { os outros; } \\
\text { Cooperar, trabalhar em } \\
\text { equipe, gerenciar e } \\
\text { resolver conflitos }\end{array}$ & $\begin{array}{l}\text { Flexibilidade e } \\
\text { adaptabilidade; } \\
\text { Iniciativa e auto } \\
\text { direcionamento; } \\
\text { Habilidades sociais e } \\
\text { multiculturais; } \\
\text { Produtividade e } \\
\text { prestação de contas; } \\
\text { Liderança e } \\
\text { responsabilidade }\end{array}$ \\
\hline Categoria & $\begin{array}{l}\text { Ferramentas para } \\
\text { trabalhar }\end{array}$ & Aprendendo a fazer & $\begin{array}{l}\text { Usar ferramentas } \\
\text { interativamente }\end{array}$ & $\begin{array}{l}\text { Ferramenta de } \\
\text { informação e } \\
\text { tecnologia }\end{array}$ \\
\hline Habilidades & $\begin{array}{l}\text { Proficiência em } \\
\text { informação; } \\
\text { Proficiência em TIC }\end{array}$ & -- & $\begin{array}{l}\text { Usar linguagens, } \\
\text { símbolos e textos } \\
\text { interativamente; } \\
\text { Usar conhecimento e } \\
\text { informação } \\
\text { interativamente; } \\
\text { Usar tecnologias } \\
\text { interativamente }\end{array}$ & $\begin{array}{l}\text { Proficiência em } \\
\text { informação; } \\
\text { Proficiência em } \\
\text { mídias; Proficiência } \\
\text { em TIC }\end{array}$ \\
\hline Categoria & Vivendo no Mundo & $\begin{array}{l}\text { Aprendendo a ser; } \\
\text { Aprendendo a viver } \\
\text { em conjunto }\end{array}$ & Agir autonomamente & $\begin{array}{l}\text { Formação geral e } \\
\text { temas para o século } \\
\text { XXI }\end{array}$ \\
\hline Habilidades & $\begin{array}{l}\text { Cidadania global e } \\
\text { local; } \\
\text { Responsabilidade } \\
\text { social e pessoal }\end{array}$ & 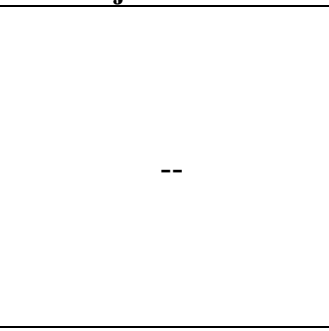 & $\begin{array}{l}\text { Agir de acordo com o } \\
\text { todo; } \\
\text { Formar e conduzir } \\
\text { planos vitalícios e } \\
\text { projetos pessoais; } \\
\text { Defender e afirmar } \\
\text { direitos, interesses, } \\
\text { limites e necessidades }\end{array}$ & $\begin{array}{l}\text { Consciência global; } \\
\text { Proficiência financeira } \\
\text { e econômica; } \\
\text { Proficiência civil; } \\
\text { Proficiência em saúde; } \\
\text { Consciência ambiental }\end{array}$ \\
\hline
\end{tabular}

Dentre esses modelos se destacam o modelo ATC21 (Binkley et al., 2011) e o modelo P21 (P21, 2015). O modelo ATC21 (Assessing and Teaching of 21st Century Skills) (Binkley et al., 2011), criado pela Cisco, Intel e Microsoft, define dez habilidades classificadas em 4 grupos, conforme apresentado na Figura 2. 


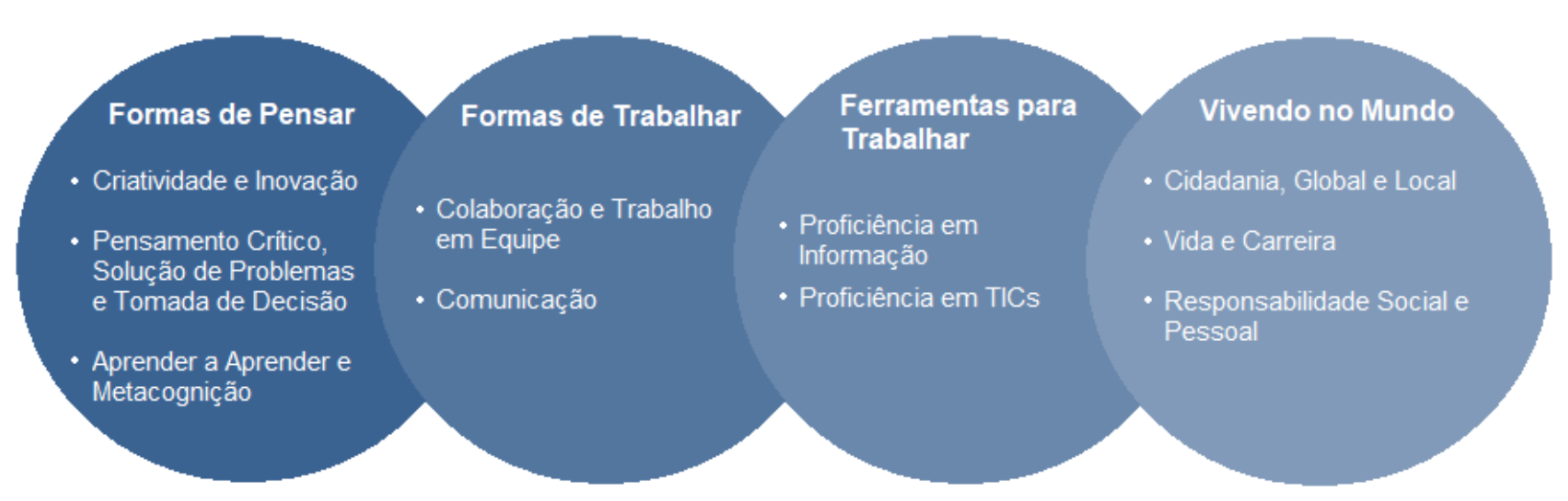

Figura 2: Habilidades do século XXI (baseado em Binkley et al, 2011).

Similar a esse modelo, o P21 (Partnership for 21st Century Learning) (P21, 2015) define dezessete habilidades separadas em quatro grupos. A maioria dessas habilidades também são definidas pelo ATC21 (Binkley et al., 2011), ressalvo alguma diferença entre os nomes das habilidades.

Ainda assim, é possível identificar algumas habilidades definidas exclusivamente pelo P21, como "Liderança", "Consciência global", "Proficiência financeira e econômica", "Proficiência econômica", "Proficiência em saúde" e "Consciência ambiental". A princípio, para o ATC21 (Binkley et al., 2011), "Liderança" é uma competência presente nas habilidades "Pensamento crítico, resolução de problemas e tomada de decisão" e "Colaboração e trabalho em equipe", já "Consciência global" é definida como um conhecimento da habilidade "Cidadania global e local". Também, a habilidade "Proficiência financeira e econômica" é descrita como parte das habilidades "Cidadania Global e local" e "Vida e carreira". Porém, não são encontradas referências significativas dentro das definições do ATC21 (Binkley et al., 2011) para as habilidades "Proficiência em saúde" e "Consciência ambiental". Em virtude disso, também foram consideradas como habilidades do século XXI as habilidades "Proficiência em Saúde" e "Consciência ambiental".

\subsection{Avaliação de Competências na Educação}

Ao longo de um ano letivo ou ao final de um curso é comum ser realizada uma avaliação com o objetivo de medir o aprendizado do aluno. Dessa maneira, uma parte essencial do processo de aprendizado é a avaliação e o feedback (Hattie \& Timperley, 2007; Shute, 2008; Black \& William, 1998; Branch, 2010). A avaliação é capaz de guiar o aprendizado do aluno e gerar feedback para o aluno e professor (Ihantola et al., 2010; Stegeman et al, 2016).

Para um aprendizado eficaz, estudantes precisam ter conhecimento do seu nível de desempenho em uma tarefa, como o seu desempenho se relaciona ao que é considerado um bom desempenho, e o que fazer para diminuir a distância entre estes (Sadler, 1989). A avaliação formativa consiste na informação comunicada para o estudante com a intenção de que ele ajuste seu pensamento/comportamento com o objetivo de aprimorar seu processo de aprendizagem (Shute, 2008). A avaliação somativa tem como objetivo providenciar ao aluno informações sobre o que eles aprenderam e qual o domínio deles sobre os conceitos aprendidos (Merrill et al., 1992; Keuning et al., 2016). Além de providenciar feedback ao aluno, a avaliação também auxilia o professor a determinar a extensão com que seus objetivos de ensino estão sendo alcançados (Ihantola et al., 2010). Além disso, a avaliação proporciona um feedback para designers instrucionais e outras partes interessadas, sendo importante para o aprimoramento contínuo de unidades instrucionais, cursos, currículos e políticas de educação. 
O uso de testes padronizados é o meio mais comum para a avaliação do aprendizado na educação básica. Uma avaliação por meio de teste consiste na avaliação do aluno de acordo com o que ele consegue resolver corretamente dado um conjunto de perguntas, tarefas ou problemas (Melhem, 2002). Vista a atual tendência de inserir o aprendizado de habilidades do século XXI na educação básica, apesar de possível a utilização de testes padronizados para a avaliação de habilidades do século XXI, como o exemplo o MSA (Mission Skills Assessment) (Petway et al., 2016). No contexto da avaliação de habilidades, o MSA utiliza uma prova de múltipla escolha para avaliar a colaboração dos estudantes perguntando a eles, por exemplo, o que eles fariam caso um membro do grupo não quisesse colaborar no projeto ao se aproximar de uma data de entrega (Soland et al, 2013). Porém, o uso de testes padronizados não necessariamente é adequado e/ou suficiente (Rusman et al., 2013; Melhem, 2002).

Neste contexto, o uso de avaliação autêntica aparenta ser o método mais apropriado para avaliar habilidades do século XXI se comparado às avaliações mais tradicionais como normativos e testes padronizados, que avaliam a habilidade de lembrar conhecimento fatual (Torrance, 1995; Ward \& Lee, 2002). Avaliações autênticas medem o desempenho do aluno baseando-se nos resultados esperados ou na observação do desempenho do aluno em atividades de aprendizado, que encorajem o uso de habilidades de alta ordem. Existem diversos tipos de testes no contexto do aprendizado de resolução de problemas, tais como avaliação de desempenho, avaliação de portfólio, autoavaliação, etc. (Brennan \& Resnick, 2012). Uma opção de teste para avaliar habilidades do século XXI pode ser a avaliação de desempenho. Este tipo de teste mede a habilidade do estudante em aplicar competências adquiridas em contextos mal estruturados ou em trabalhar em equipe para solucionar problemas complexos (Wiggins, 1993).

Outra alternativa é a autoavaliação por meio de questionários, amplamente suportada pela ciência psicométrica, que utiliza questionários para captar as percepções dos estudantes em uma variedade de áreas (Devellis, 2016). A autoavaliação é bem aceita para medir diversos fatores, mas, apesar de possibilitar a coleta de dados válidos (Sitzmann et al., 2010; Topping, 2003; Ross, 2006), pode ser tendencioso e não confiável, gerando controvérsia quanto aos seus usos (Ross, 2006). Por isso, o uso de instrumentos de coleta de dados padronizados é importante para reduzir essas possíveis ameaças à sua validade. Dessa maneira, para contribuir de forma efetiva na melhoria do ensino da computação e da sua contribuição para o aprendizado de habilidades do século XXI é essencial que o modelo e os seus instrumentos de coleta de dados sejam desenvolvidos e avaliados sistematicamente.

\section{Trabalhos Relacionados}

Analisando o estado da arte por meio de um mapeamento sistemático da literatura (Autores, 2018), foram encontrados oito modelos de avaliação de habilidades do século XXI (Chai et al., 2015; Rosen, 2015; Siddiq et al., 2017; Claro et al., 2012; Rosen \& Tager, 2014; Aesaert et al., 2014; Lau \& Yuen, 2014; Susnea \& Vasiliu, 2016). Observa-se que os modelos encontrados são recentes, publicados a partir de 2012, indicando a importância e o interesse em avaliações de habilidades do século XXI nos últimos anos.

Os modelos de avaliação encontrados propõem soluções para avaliar habilidades, porém, sempre de maneira isolada, por habilidade ou por grupo. Nenhum dos modelos se propôs a avaliar todas as habilidades do século XXI conforme definido pela Binkley (2011) e ou do modelo P21 (P21, 2015). Observando uma variação também entre as habilidades avaliadas por cada um dos modelos, evidencia-se a falta de um consenso de como avaliar, e até a própria terminologia/definição das habilidades do século XXI. Entre as habilidades avaliadas, existe uma preocupação maior em avaliar habilidades referentes ao uso de tecnologias de informação. 
Observa-se que a habilidade "Proficiência em TIC" recebe maior atenção, sendo a habilidade mais avaliada pelos modelos. Referente ao modelo ICTSfL (Claro et al., 2012), os autores ressaltam o quão importante são essas habilidades, e como elas podem englobar e ajudar a desenvolver outras habilidades como Comunicação, Colaboração \& Trabalho em Equipe e Responsabilidade Pessoal \& Social.

A maioria dos modelos encontrados realiza a coleta de dados para a avaliação por meio de testes (Rosen, 2015; Siddiq et al., 2017; Claro et al., 2012; Rosen \& Tager, 2014; Aesaert et al., 2014). Estes testes muitas vezes são realizados em formato digital, utilizando um software, geralmente desenvolvido pelos próprios autores, salvo um modelo que utilizou uma adaptação de um software existente. Outros modelos (Chai et al., 2015; Lau \& Yuen, 2014; Susnea \& Vasiliu, 2016) utilizaram autoavaliações por meio de questionário para a coleta de dados. Um modelo (Siddiq et al., 2017) utilizou juntamente com o teste, um questionário socioeconômico, possibilitando o relacionamento do perfil socioeconômico com o desempenho no teste. Outro modelo (Rosen, 2015) também se destacou por utilizar, também junto com o teste, um questionário de feedback, em que os alunos puderam expressar e avaliar a habilidade de colaboração dos seus colegas de equipe.

A maioria dos instrumentos propostos foi avaliada usando diversas técnicas estatísticas, tais como coeficiente alfa de Cronbach e análise da correlação entre itens. Alguns, como o modelo de Rosen e Tager (2014), foram validados somente por um painel de especialistas e representantes do público-alvo. Porém, todos os instrumentos foram validados, o que demonstra preocupação com a qualidade dos modelos utilizados para avaliar habilidades do século XXI.

Observa-se ainda, como um dos principais pontos fracos, a falta de cobertura mais completa de habilidades sendo avaliados em relação aos frameworks/modelos de definição de habilidades do século XXI, permitindo assim uma avaliação das habilidades de maneira mais abrangente em conformidade com os principais frameworks/modelos.

\section{Método de Pesquisa}

Com o objetivo de desenvolver e avaliar o modelo bASES21, uma pesquisa multimétodo é adotada, conforme apresenta na Figura 3. 


\begin{tabular}{|c|c|c|c|c|}
\hline \multirow[t]{2}{*}{ Entradas } & \multicolumn{2}{|c|}{$\begin{array}{l}\text { Análise do estado da arte de modelos de avaliação de } \\
\text { habilidades do século XXI no contexto de ensino de } \\
\text { computação na educação básica. } \\
\text { (Autores, 2018). }\end{array}$} & & \\
\hline & $\begin{array}{c}\begin{array}{c}\text { Framework P21 } \\
(\mathrm{P} 21,2015)\end{array} \\
\end{array}$ & $\begin{array}{l}\text { Framework ACTS21 } \\
\text { (Binkley et al., 2011) } \\
\end{array}$ & & \\
\hline Etapas & Atividades & & Métodos & Resultados \\
\hline \multirow{10}{*}{$\begin{array}{l}\text { Etapa } 1 . \\
\text { Desenvolvimento do } \\
\text { modelo bASE21 }\end{array}$} & \multirow{2}{*}{\multicolumn{2}{|c|}{ Definir o objetivo da avaliação }} & & \\
\hline & & & & \\
\hline & \multicolumn{2}{|c|}{$\boldsymbol{v}$} & $\mathrm{GQM}$ & \\
\hline & \multicolumn{2}{|c|}{ Definir as perguntas de análise e medidas } & (Basili et al, 1994) & \\
\hline & \multicolumn{2}{|c|}{$\downarrow$} & Guia de Design de & \\
\hline & \multicolumn{2}{|c|}{ Definir o instrumento de coleta de dados } & Questionários & Modelo bASE2 1 v0.9 \\
\hline & \multicolumn{2}{|c|}{$\downarrow$} & (Kasunic, 2005) & \\
\hline & \multicolumn{2}{|c|}{ Definir o formato de respostas } & Painel de Especialistas & \\
\hline & \multicolumn{2}{|c|}{$\boldsymbol{\psi}$} & (Beecham et al, 2005) & \\
\hline & \multicolumn{2}{|c|}{ Avaliar a validade aparente } & & \\
\hline \multirow{6}{*}{$\begin{array}{l}\text { Etapa } 2 . \\
\text { Avaliação do modelo } \\
\text { bASE21 }\end{array}$} & \multicolumn{2}{|c|}{$\downarrow$} & & \\
\hline & \multicolumn{2}{|c|}{ Definir a avaliação } & $\begin{array}{l}\text { Estudos de caso } \\
\text { (Wohlin et al., 2012) }\end{array}$ & Confiabilidadee \\
\hline & \multicolumn{2}{|c|}{$\downarrow$} & GQM & bASE21 \\
\hline & \multicolumn{2}{|c|}{ Executar a avaliação } & & \\
\hline & \multicolumn{2}{|c|}{$\downarrow$} & $\begin{array}{l}\text { - Alfa de Cronbach } \\
\text { - Correlação de itens }\end{array}$ & \\
\hline & \multicolumn{2}{|c|}{ Analisar os dados } & $\begin{array}{c}\text { (Devellis, 2016) } \\
\text { (Trochim; Donnelly; 2008) }\end{array}$ & Modelo bASE21 v1.0 \\
\hline
\end{tabular}

Figura 3: Método de pesquisa.

Como uma etapa anterior a este estudo, foi realizado um mapeamento sistemático da literatura com o objetivo de elicitar o estado da arte em relação a modelos de avaliação de habilidades do século XXI, identificando quais modelos existem e quais as suas características, considerando o contexto de ensino de computação no ensino básico (Autores, 2018). São utilizados também como entrada frameworks/modelos existentes que definem sistematicamente as habilidades do século XXI, como P21 e ATC21.

Etapa 1. Desenvolvimento do modelo bASE21. Nesta etapa o modelo bASE21 é desenvolvido, levando em consideração os resultados do levantamento do estado da arte e com base nos frameworks/modelos existentes. O desenvolvimento do modelo bASE21 segue o método Goal/Question/Metric (GQM) (Basili et al., 1994) e os procedimentos do guia de design de questionários proposto por Kasunic (2005):

Definir o objetivo da avaliação. Com base na abordagem GQM o objetivo da avaliação é definido.

Definir as perguntas de análise e medidas. A partir do objetivo definido, seguindo a abordagem GQM, o mesmo é decomposto sistematicamente em perguntas de análise e medidas, considerando os resultados do levantamento do estado da arte e a definição das habilidades do século XXI dos frameworks/modelos existentes.

Definir o instrumento de coleta de dados. Para sistematicamente coletar dados sobre as medidas definidas, um instrumento de coleta de dados é definido seguindo o guia proposto por Kasunic (2005). Para cada uma das medidas, itens são derivados com base também na literatura existente.

Definir o formato de respostas. Nesta etapa, o formato de resposta para os itens do instrumento de coleta de dados é definido. Esta definição é baseada em formatos de resposta 
tipicamente usados em questionários padronizados e seguindo o guia de design de questionários proposto por Kasunic (2005).

Avaliar a validade aparente. Nesta etapa é realizada a análise do instrumento de coleta de dados por um painel de especialistas (Beecham et al., 2005). A análise pelos especialistas objetiva revisar o questionário em termos de sua completude e consistência e a relevância e compreensão dos itens. O painel é composto por especialistas das áreas de computação, educação, design e microeletrônica, e representantes do público alvo (crianças e jovens da educação básica).

Etapa 2. Avaliação do modelo bASE21. Nesta etapa, o modelo desenvolvido é avaliado em termos de confiabilidade e validade pela realização de um estudo de caso (Wohlin et al., 2012), sendo aplicado na educação básica.

Definir a avaliação. O objetivo da avaliação do modelo é definido seguindo a abordagem GQM (Basili et al., 1994) e decomposto em aspectos de qualidade e perguntas de análise.

Executar a avaliação. Nesta etapa, o estudo de caso é conduzido com alunos da educação básica. Os dados são coletados a partir da aplicação do questionário do modelo bASE21 em um estudo de caso one-shot na educação básica (sem aplicação de um tratamento específico). Os dados são coletados por meio do preenchimento do questionário, disponibilizado de maneira impressa ou online, por estudantes de diferentes turmas da educação básica.

Analisar os dados. Os dados coletados são agrupados e analisados de forma estatística de modo a identificar a validade e confiabilidade (Trochim \& Donnelly, 2008) do instrumento de medição do modelo bASE21.

\section{Desenvolvimento do Modelo bASE21}

O objetivo do modelo bASES21 (Assessing $21^{\text {st }}$ CEntury Skills) é avaliar as habilidades do século XXI do aluno no contexto do ensino da computação na educação básica a partir da percepção do aluno. O modelo é baseado na definição de habilidades do século XXI dos frameworks ATC21 (Binkley et al., 2010) e P21 (2015). Também tendo em vista o contexto da aplicação do modelo no ensino da computação, foi adicionada a habilidade "Proficiência em computação", baseada no K-12 Computer Science Framework (2016), visando a importância da habilidade de computação como também uma das habilidades importantes no século XXI (CSF, 2016). A Tabela 3 apresenta as definições das habilidades do século XXI abordadas pelo modelo bASE21.

Tabela 3: Definição das habilidades do Século XXI abordadas pelo modelo bASES21.

\begin{tabular}{|c|c|c|}
\hline Habilidade & Descrição & Fonte \\
\hline Criatividade e inovação & $\begin{array}{l}\text { Criar novas ideias que sejam válidas ou inovadoras. Desenvolver, } \\
\text { refinar e comunicar eficientemente novas ideias. }\end{array}$ & $\begin{array}{l}\text { (Binkley et al., } \\
\text { 2011) }\end{array}$ \\
\hline $\begin{array}{l}\text { Pensamento crítico, } \\
\text { resolução de problemas e } \\
\text { tomada de decisão }\end{array}$ & $\begin{array}{l}\text { Usar o tipo de raciocínio adequado para cada situação. Elaborar } \\
\text { argumentos e soluções a partir da análise de evidências e } \\
\text { experiências. Tomar decisões baseando-se na melhor } \\
\text { análise/interpretação de informações. }\end{array}$ & $\begin{array}{l}\text { (Binkley et al., } \\
\text { 2011) }\end{array}$ \\
\hline $\begin{array}{l}\text { Aprender a aprender e } \\
\text { metacognição }\end{array}$ & $\begin{array}{l}\text { Compreender métodos de aprendizado que funcionam para si. } \\
\text { Perceber pontos fortes e fracos em relação ao próprio } \\
\text { aprendizado. }\end{array}$ & $\begin{array}{l}\text { (Binkley et al., } \\
\text { 2011) }\end{array}$ \\
\hline Comunicação & $\begin{array}{l}\text { Comunicar-se de maneira clara e eficiente. Usar diferentes meios } \\
\text { de comunicação, interações verbais e vocabulários de acordo com } \\
\text { o contexto da comunicação. }\end{array}$ & $\begin{array}{l}\text { (Binkley et al., } \\
\text { 2011) }\end{array}$ \\
\hline Colaboração e trabalho em & Saber distinguir os momentos de fala e de atenção. Participar em & (Binkley et al., \\
\hline
\end{tabular}




\begin{tabular}{|c|c|c|}
\hline equipe & $\begin{array}{l}\text { trabalho em equipe de maneira harmoniosa e respeitosa com os } \\
\text { outros membros da equipe. }\end{array}$ & 2011) \\
\hline Proficiência em informação & $\begin{array}{l}\text { Saber buscar, usar, refinar e extrair informações de diferentes } \\
\text { fontes. Distinguir informações relevantes de informações não } \\
\text { relevantes. }\end{array}$ & $\begin{array}{l}\text { (Binkley et al., } \\
\text { 2011) }\end{array}$ \\
\hline Proficiência em TIC & $\begin{array}{l}\text { Conhecer e saber usar as principais aplicações de informação e } \\
\text { comunicação disponíveis em um computador e por meio da } \\
\text { internet. }\end{array}$ & $\begin{array}{l}\text { (Binkley et al., } \\
\text { 2011) }\end{array}$ \\
\hline Cidadania global e local & $\begin{array}{l}\text { Compreender os direitos de um cidadão, os papéis das } \\
\text { instituições públicas e o conceito de comunidades. Participar nas } \\
\text { atividades da comunidade local e internacional, atuando nas } \\
\text { tomadas de decisões e resolução de problemas. }\end{array}$ & $\begin{array}{l}\text { (Binkley et al., } \\
\text { 2011) }\end{array}$ \\
\hline Vida e carreira & $\begin{array}{l}\text { Estar consciente da volatilidade e da importância das } \\
\text { oportunidades geradas pelo ambiente ao seu redor. Atuar em } \\
\text { diferentes trabalhos, responsabilidades e contextos. }\end{array}$ & $\begin{array}{l}\text { (Binkley et al., } \\
\text { 2011) }\end{array}$ \\
\hline $\begin{array}{l}\text { Responsabilidade social e } \\
\text { pessoal }\end{array}$ & $\begin{array}{l}\text { Respeitar diferentes culturas, superar estereótipos e preconceitos. } \\
\text { Demonstrar empatia e interesse pela opinião dos outros. }\end{array}$ & $\begin{array}{l}\text { (Binkley et al., } \\
\text { 2011) }\end{array}$ \\
\hline Proficiência em computação & $\begin{array}{l}\text { Interagir com computadores e saber utilizá-los para realizar } \\
\text { tarefas. Entender o potencial e o impacto da computação na } \\
\text { sociedade. Utilizar o pensamento computacional para resolver } \\
\text { problemas. }\end{array}$ & (CSTA, 2017) \\
\hline Proficiência em saúde & $\begin{array}{l}\text { Saber como manter a saúde mental e física de uma pessoa, por } \\
\text { meio de uma dieta saudável, prática de exercícios, diminuir riscos } \\
\text { e níveis de stress. }\end{array}$ & $(\mathrm{P} 21,2017)$ \\
\hline Consciência ambiental & $\begin{array}{l}\text { Demonstrar interesse em questões ambientais e tomar atitudes } \\
\text { pessoais e coletivas voltadas para a preservação e recuperação } \\
\text { ambiental. Saber sobre os impactos da sociedade no meio } \\
\text { ambiente. }\end{array}$ & $(\mathrm{P} 21,2017)$ \\
\hline
\end{tabular}

A partir das habilidades identificadas é definida uma pergunta de análise para cada habilidade (Tabela 4).

Tabela 4: Perguntas de análise do modelo bASES21.

\begin{tabular}{|c|l|}
\hline \multicolumn{1}{|c|}{ ID } & \multicolumn{1}{c|}{ Perguntas de análise } \\
\hline PA1 & Qual o grau da habilidade Criatividade e inovação? \\
\hline PA2 & Qual o grau da habilidade Pensamento crítico, resolução de problemas e tomada de decisão? \\
\hline PA3 & Qual o grau da habilidade Aprender a aprender e metacognição? \\
\hline PA4 & Qual o grau da habilidade Comunicação? \\
\hline PA5 & Qual o grau da habilidade Colaboração e trabalho em equipe? \\
\hline PA6 & Qual o grau da habilidade Proficiência em informação? \\
\hline PA7 & Qual o grau da habilidade Proficiência em TIC? \\
\hline PA8 & Qual o grau da habilidade Cidadania global e local? \\
\hline PA9 & Qual o grau da habilidade Vida e carreira? \\
\hline PA10 & Qual o grau da habilidade Responsabilidade social e pessoal? \\
\hline PA11 & Qual o grau da habilidade Proficiência em computação? \\
\hline PA12 & Qual o grau da habilidade Proficiência em saúde? \\
\hline PA13 & Qual o grau da habilidade Consciência ambiental? \\
\hline
\end{tabular}

O público alvo do modelo de avaliação são estudantes da educação básica brasileira (estudantes do ensino fundamental e ensino médio). A aplicação do modelo pode ser feita de diferentes formas, dependendo do tipo do estudo e do research design escolhido. O modelo pode ser aplicado em estudos não experimentais, utilizando one-shot post-test designs com aplicações pontuais e/ou depois do tratamento (e.g. o ensino de computação) ou one-shot pretest/post-test antes e depois do tratamento. O modelo também pode ser utilizado em estudos (quase-)experimentais utilizando somente post-test ou pre-test/post-test designs, envolvendo grupos de controle (realizando outro tipo de tratamento, ou nenhum tratamento). 
A partir das perguntas de análise definidas é desenvolvido como instrumento de coleta de dados um questionário de autoavaliação. Optou-se por essa forma de avaliação, nesse ponto inicial da pesquisa, por representar uma forma de avaliação não interruptiva ao fluxo normal de uma aula e que não prejudique o público-alvo envolvido na pesquisa. Embora não há consenso na literatura, há evidências de que a autoavaliação produz dados confiáveis, válidos e úteis (Topping, 2003; Ross, 2006), especialmente quando se utiliza instrumentos de medição confiáveis e válidos (Sitzmann et al., 2010). Portanto, um compromisso pode ser o desenvolvimento de instrumentos de medição padronizados e estatisticamente validados, aumentando a validade e a confiabilidade dos dados coletados na autoavaliação (Kasunic, 2005; Devellis, 2016).

Os itens do questionário são definidos com base na literatura encontrada e também com base nos modelos ATC21 (Binkley et al., 2011) e P21 (2017). Quanto às habilidades que não foram contempladas por nenhum dos modelos encontrados, o desenvolvimento dos itens é baseado em referências complementares. A formulação dos itens foi feita cuidadosamente, levando em consideração o público alvo, de maneira que todos os itens sejam corretamente compreendidos, e respondidos, mantendo a concordância das respostas dos itens com a escala.

É definida como formato de resposta uma escala ordinal de quatro pontos: "Discordo totalmente", "Discordo", "Concordo" e "Concordo totalmente". A escala de quatro pontos é mais utilizada para os casos em que é importante que o respondente tome um posicionamento, seja ele qual for, quanto ao item (Losby \& Wetmore, 2012). Apesar de forçar o estudante a pensar mais sobre a sua resposta, também é dada a opção de deixar o item em branco, caso o aluno não deseje responder o item.

A versão preliminar do questionário foi revisada por um painel de especialistas. O painel foi composto por 9 especialistas das áreas de computação, educação, design e microeletrônica, e 3 representantes do público alvo (crianças/jovens de 11 a 15 anos). Nesta revisão os especialistas revisaram cada item do questionário em relação a sua relevância e compreensão. O questionário também foi avaliado em termos de sua completude e consistência de acordo com as habilidades que ele propõe avaliar. A partir do feedback obtido, vários itens do questionário foram alterados, principalmente em relação a sua formulação, com o objetivo de melhorar a compreensão dos itens pelo público alvo. Dois itens, relacionados as habilidades "Colaboração e trabalho em equipe" foram unificados pois eram referentes a assuntos muito próximos. Um item foi adicionado a habilidade "Proficiência em computação" por representar um assunto importante para a habilidade. Nenhum item foi excluído do questionário. Como resultado dessa primeira revisão foi definida a versão 0.9 do questionário com 82 itens, apresentado na Tabela 5.

Tabela 5: Versão 0.9 do questionário de autoavaliação do modelo bASES21.

\begin{tabular}{|l|c|l|l|}
\hline \multicolumn{1}{|c|}{ Habilidade } & ID & \multicolumn{1}{|c|}{ Item } & \multicolumn{1}{c|}{ Fonte/Referência } \\
\hline $\begin{array}{l}\text { Criatividade e } \\
\text { inovação }\end{array}$ & 1 & $\begin{array}{l}\text { Eu invento/imagino muitas coisas que ainda } \\
\text { não existem }\end{array}$ & $\begin{array}{l}\text { I generate many new ideas (Chai et al., 2015); I generate novel ideas } \\
\text { (Petway et al., 2016); I have lots of ideas in every domain (Susnea \& } \\
\text { Vasiliu, 2016) }\end{array}$ \\
\cline { 2 - 4 } & 2 & Minhas ideias são úteis & I produce ideas that are likely to be useful (Chai et al., 2015) \\
\cline { 2 - 5 } & 3 & $\begin{array}{l}\text { Eu consigo resolver um problema de } \\
\text { maneiras diferentes }\end{array}$ & $\begin{array}{l}\text { I create different solutions for a problem (Chai et al., 2015); I suggest } \\
\text { new ways of doing things (Chai et al., 2015); I provide solutions that } \\
\text { no one else thought of (Kang et al., 2010) }\end{array}$ \\
\cline { 2 - 5 } & 4 & Eu sou uma pessoa curiosa & I am very curious (Susnea \& Vasiliu, 2016); \\
\cline { 2 - 5 } & 5 & $\begin{array}{l}\text { Não tenho vergonha de falar sobre as } \\
\text { minhas ideias }\end{array}$ & $\begin{array}{l}\text { I feel very embarrassed if I fail (escala inversa, Susnea \& Vasiliu, } \\
\text { 2016) }\end{array}$ \\
\cline { 2 - 5 } & 6 & $\begin{array}{l}\text { Eu aprendo com os meus erros ou quando } \\
\text { minhas ideias dão errado }\end{array}$ & $\begin{array}{l}\text { View failure as an opportunity to learn; understand that creativity and } \\
\text { innovation is a long-term, cyclical process of small successes and } \\
\text { frequent mistakes (Binkley et al., 2011) }\end{array}$ \\
\cline { 2 - 5 } & 7 & Eu tento melhorar minhas ideias & $\begin{array}{l}\text { Elaborate, refine, analyze and evaluate their own ideas to improve and } \\
\text { maximize creative efforts (P21, 2015) }\end{array}$ \\
\hline
\end{tabular}




\begin{tabular}{|c|c|c|c|}
\hline \multirow{9}{*}{$\begin{array}{l}\text { Pensamento crítico, } \\
\text { resolução de } \\
\text { problemas e } \\
\text { tomada de decisão }\end{array}$} & 8 & $\begin{array}{l}\text { Eu comparo opiniões/ideias diferentes para } \\
\text { ver qual é a melhor }\end{array}$ & $\begin{array}{l}\text { I consider different opinions to see which one makes more sense (Chai } \\
\text { et al., 2015); Consider and evaluate major alternative points of view } \\
\text { (Binkley et al., 2011) }\end{array}$ \\
\hline & 9 & $\begin{array}{l}\text { Eu tomo decisões de acordo com as } \\
\text { informações que eu tenho }\end{array}$ & $\begin{array}{l}\text { Interpret information and draw conclusions based on the best analysis. } \\
\text { Categorize, decode and clarify information (Binkley et al., 2011) }\end{array}$ \\
\hline & 10 & $\begin{array}{l}\text { Eu gosto de fazer e responder perguntas para } \\
\text { aprender algo novo }\end{array}$ & $\begin{array}{l}\text { I usually raise questions on ordinary thoughts and look for alternatives } \\
\text { (Kang et al., 2010); Ask significant questions that clarify various } \\
\text { points of view and lead to better solutions. Clearly articulate the results } \\
\text { of one's inquiry (Binkley et al., 2011); Asks and answers question to } \\
\text { deepen understanding (IFL, 2015) }\end{array}$ \\
\hline & 11 & $\begin{array}{l}\text { Eu ouço as ideias dos meus colegas e as } \\
\text { considero quando formo minha opinião }\end{array}$ & $\begin{array}{l}\text { Consider and evaluate major alternative points of view. Reflect } \\
\text { critically on learning experiences and processes. Incorporate these } \\
\text { reflections into the decision-making process (Binkley et al., 2011) }\end{array}$ \\
\hline & 12 & $\begin{array}{l}\text { Eu tento entender um problema antes de } \\
\text { tentar resolve-lo }\end{array}$ & $\begin{array}{l}\text { I try to understand tasks before I attempt to solve them (O'Niel \& } \\
\text { Schacter, 1997) }\end{array}$ \\
\hline & 13 & $\begin{array}{l}\text { Eu escolho e organizo o material que preciso } \\
\text { quando vou fazer algo (tarefas de casa, } \\
\text { trabalhos, estudar, etc.) }\end{array}$ & $\begin{array}{l}\text { I select and organize relevant information to solve a task (O'niel \& } \\
\text { Schacter, 1997) }\end{array}$ \\
\hline & 14 & $\begin{array}{l}\text { Eu me pergunto se estou fazendo bem as } \\
\text { minhas tarefas da escola }\end{array}$ & $\begin{array}{l}\text { I ask myself, how well am I doing, as I proceed through tasks (O'niel } \\
\text { \& Schacter, 1997) }\end{array}$ \\
\hline & 15 & $\begin{array}{l}\text { Eu me esforço quando faço as minhas } \\
\text { tarefas da escola }\end{array}$ & I work as hard as possible on tasks (O'niel \& Schacter, 1997) \\
\hline & 16 & $\begin{array}{l}\text { Eu consigo explicar as minhas opiniões e } \\
\text { decisões }\end{array}$ & I provide reasons and evidences for my opinions (Chai et al., 2015) \\
\hline \multirow[t]{5}{*}{$\begin{array}{l}\text { Aprender a } \\
\text { aprender e } \\
\text { metacognição }\end{array}$} & 17 & $\begin{array}{l}\text { Eu planejo como vou estudar (quais } \\
\text { exercícios vou fazer em que dias/tempo, } \\
\text { etc.) }\end{array}$ & I make plans for how I will study (Chai et al., 2015) \\
\hline & 18 & $\begin{array}{l}\text { Se estou tendo dificuldade em um assunto } \\
\text { da matéria eu dedico mais tempo de estudo } \\
\text { para esse assunto }\end{array}$ & I adjust the ways I study based on my progression (Chai et al., 2015) \\
\hline & 19 & $\begin{array}{l}\text { Acredito que consigo aprender tudo que } \\
\text { quiser }\end{array}$ & $\begin{array}{l}\text { A self-concept that supports a willingness to change and further } \\
\text { develop skills as well as self-motivation and confidence in one's } \\
\text { capability to succeed (Binkley et al., 2011) }\end{array}$ \\
\hline & 20 & Eu gosto de aprender coisas novas & $\begin{array}{l}\text { Positive appreciation of learning as a life-enriching activity and a sense } \\
\text { of initiative to learn (Binkley et al., 2011) }\end{array}$ \\
\hline & 21 & $\begin{array}{l}\text { Eu consigo me manter concentrado(a) por } \\
\text { muito tempo }\end{array}$ & $\begin{array}{l}\text { Ability to concentrate for extended as well as short periods of time } \\
\text { (Binkley et al., 2011) }\end{array}$ \\
\hline \multirow[t]{6}{*}{ Comunicação } & 22 & $\begin{array}{l}\text { Eu ouço com atenção para entender o que os } \\
\text { outros falam }\end{array}$ & $\begin{array}{l}\text { Ability to listen to and understand various spoken messages in a } \\
\text { variety of communicative situations and to speak concisely and clearly } \\
\text { (Binkley et al., 2011); }\end{array}$ \\
\hline & 23 & Outras pessoas entendem o que eu falo & $\begin{array}{l}\text { Ability to communicate, in written or oral form, and understand, or } \\
\text { make others understand, various messages in a variety of situations and } \\
\text { for different purposes (Binkley et al., 2011) }\end{array}$ \\
\hline & 24 & $\begin{array}{l}\text { Quando eu leio um texto, eu entendo sobre o } \\
\text { que estou lendo }\end{array}$ & $\begin{array}{l}\text { Ability to read and understand different texts, adopting strategies } \\
\text { appropriate to various reading purposes (reading for information, for } \\
\text { study or for pleasure) and to various text types (Binkley et al., 2011) }\end{array}$ \\
\hline & 25 & Não tenho vergonha de falar em público & Confidence when speaking in public (Binkley et al., 2011) \\
\hline & 26 & $\begin{array}{l}\text { Gosto de conversar e ouvir opiniões } \\
\text { diferentes }\end{array}$ & $\begin{array}{l}\text { Disposition to approach the opinions and arguments of others with an } \\
\text { open mind and engage in constructive and critical dialogue (Binkley et } \\
\text { al., 2011) }\end{array}$ \\
\hline & 27 & $\begin{array}{l}\text { Eu consigo fazer bons argumentos em um } \\
\text { debate }\end{array}$ & $\begin{array}{l}\text { Ability to formulate one's arguments, in speaking or writing, in a } \\
\text { convincing manner and take full account of other viewpoints, whether } \\
\text { expressed in written or oral form (Binkley et al., 2011) }\end{array}$ \\
\hline
\end{tabular}




\begin{tabular}{|c|c|c|c|}
\hline \multirow[t]{11}{*}{$\begin{array}{l}\text { Colaboração e } \\
\text { trabalho em equipe }\end{array}$} & 28 & $\begin{array}{l}\text { Eu gosto de trabalhar junto com os meus } \\
\text { colegas para fazer trabalhos ou resolver } \\
\text { problemas }\end{array}$ & $\begin{array}{l}\text { My classmates and I actively work together to complete tasks (Chai et } \\
\text { al., 2015); I like to work with people (Kyllonen, 2012; Petway et al., } \\
\text { 2016); I cooperate with other students (Petway et al., 2016); I usually } \\
\text { cooperate and work well with others (Kang et al., 2010) }\end{array}$ \\
\hline & 29 & $\begin{array}{l}\text { Eu consigo arranjar um tempo para ajudar } \\
\text { outras pessoas }\end{array}$ & I think it is important to help people (Kyllonen, 2012) \\
\hline & 30 & Eu gosto de ser o(a) líder do grupo & $\begin{array}{l}\text { I like leading groups (Kyllonen, 2012); I try to be a leader in a group } \\
\text { learning situation (Kang et al., 2010) }\end{array}$ \\
\hline & 31 & $\begin{array}{l}\text { Eu sempre faço a minha parte quando } \\
\text { trabalho em grupo }\end{array}$ & $\begin{array}{l}\text { I try my best to perform my role in a group learning situation (Kang et } \\
\text { al., 2010); I am usually reliable in a group learning situation (Kang et } \\
\text { al., 2010) }\end{array}$ \\
\hline & 32 & $\begin{array}{l}\text { Eu consigo criar uma sequência para as } \\
\text { tarefas de um trabalho em grupo }\end{array}$ & $\begin{array}{l}\text { Prioritize, plan and manage work to achieve the intended group result } \\
\text { (Binkley et al., 2011) }\end{array}$ \\
\hline & 33 & $\begin{array}{l}\text { Eu gosto de ser um bom exemplo para os } \\
\text { outros }\end{array}$ & $\begin{array}{l}\text { Inspire others to reach their very best via example and selflessness } \\
\text { (Binkley et al., 2011); }\end{array}$ \\
\hline & 34 & $\begin{array}{l}\text { Eu respeito as diferenças das pessoas de } \\
\text { outras regiões, países e religiões. }\end{array}$ & $\begin{array}{l}\text { Show respect for cultural differences and be prepared to work } \\
\text { effectively with people from a range of social and cultural } \\
\text { backgrounds (Binkley et al., 2011) }\end{array}$ \\
\hline & 35 & $\begin{array}{l}\text { Eu me comprometo a fazer as tarefas } \\
\text { necessárias para atingir o objetivo de um } \\
\text { trabalho em grupo }\end{array}$ & $\begin{array}{l}\text { Exercise flexibility and willingness to be helpful in making necessary } \\
\text { compromises to accomplish a common goal (P21, 2015) }\end{array}$ \\
\hline & 36 & $\begin{array}{l}\text { Em um trabalho em grupo, geralmente meus } \\
\text { colegas concordam com as minhas decisões }\end{array}$ & $\begin{array}{l}\text { In a situation where we need to make decisions together, my friends } \\
\text { usually follow my choice (Kang et al., 2010) }\end{array}$ \\
\hline & 37 & Eu não desisto facilmente & $\begin{array}{l}\text { I give up easily (escala inversa, Kyllonen, 2012); Sometimes I get } \\
\text { obsessed with a problem, and I keep trying until I find a solution } \\
\text { (Susnea \& Vasiliu, 2016) }\end{array}$ \\
\hline & 38 & Eu geralmente termino as coisas que começo & I finish whatever I begin (Duckworth et al., 2007) \\
\hline \multirow[t]{7}{*}{$\begin{array}{l}\text { Proficiência em } \\
\text { Informação }\end{array}$} & 39 & $\begin{array}{l}\text { Eu consigo encontrar as informações } \\
\text { necessárias para fazer um trabalho/resolver } \\
\text { um problema }\end{array}$ & $\begin{array}{l}\text { I can identify appropriately the needed information from question (Lau } \\
\text { \& Yuen, 2014); Pupils can assess and judge the relevance of the } \\
\text { information that was found for answering a question (Aesaert et al., } \\
\text { 2014); Propensity to use information to work autonomously and in } \\
\text { teams (Binkley et al., 2011); I can locate and make use of data or } \\
\text { information that are helpful to my studies (Kang et al., 2010) }\end{array}$ \\
\hline & 40 & $\begin{array}{l}\text { Eu analiso se uma informação é confiável ou } \\
\text { não }\end{array}$ & 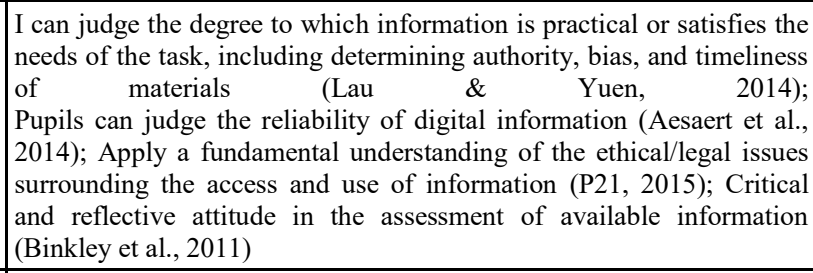 \\
\hline & 41 & $\begin{array}{l}\text { Eu posso mudar de opinião dependendo de } \\
\text { quanto eu sei sobre o assunto }\end{array}$ & $\begin{array}{l}\text { Improvement or changed - based on team responses (Siddiq et al., } \\
\text { 2017) }\end{array}$ \\
\hline & 42 & $\begin{array}{l}\text { Eu consigo explicar porque mudei de } \\
\text { opinião }\end{array}$ & Reasoning/explain why you changed your answer (Siddiq et al., 2017) \\
\hline & 43 & Eu consigo interpretar gráficos e tabelas & $\begin{array}{l}\text { The student had to read and analyze different kinds of information } \\
\text { (graphs, tables, texts), and then to choose the best interpretation for the } \\
\text { information among five alternatives (Claro et al., 2012); Ability to use } \\
\text { appropriate aids (presentations, graphs, charts, maps) to produce, } \\
\text { present or understand complex information (Binkley et al., 2011) }\end{array}$ \\
\hline & 44 & $\begin{array}{l}\text { Considero errado copiar, compartilhar ou } \\
\text { alterar coisas (informação, texto, fotos, etc.) } \\
\text { de outras pessoas sem a permissão delas }\end{array}$ & $\begin{array}{l}\text { Apply a fundamental understanding of the ethical/legal issues } \\
\text { surrounding the access and use of information/media }(\mathrm{P} 21,2015)\end{array}$ \\
\hline & 45 & $\begin{array}{l}\text { Quando eu estudo, eu busco mais } \\
\text { informações além das anotações do meu } \\
\text { caderno ou apostila/livro }\end{array}$ & $\begin{array}{l}\text { When I study, I try to find answers to my questions (Kang et al., 2010); } \\
\text { When I study, I look for answers on the Internet or in the library (Kang } \\
\text { et al., 2010) }\end{array}$ \\
\hline
\end{tabular}




\begin{tabular}{|c|c|c|c|}
\hline \multirow[t]{5}{*}{$\begin{array}{l}\text { Proficiência em } \\
\text { TIC }\end{array}$} & 46 & $\begin{array}{l}\text { Quando eu estudo eu uso a internet para } \\
\text { achar informações úteis }\end{array}$ & $\begin{array}{l}\text { Pupils can use a search engine by entering one or more correct search } \\
\text { terms derived from a task or question (Aesaert et al., 2014); I find out } \\
\text { useful information on the Internet to help my learning (Chai et al., } \\
\text { 2015); When I study, I look for answers on the Internet or in the library } \\
\text { (Kang et al., 2010); I can search for information on the internet using a } \\
\text { search engine (e.g. Yahoo, Google, Baidu) (Lau \& Yuen, 2014); Use } \\
\text { various data collection techniques for different types of problems (e.g., } \\
\text { mobile device GPS, user survey, embedded system sensors, open data } \\
\text { sets, social media data sets, etc.) (CSTA, 2017) }\end{array}$ \\
\hline & 47 & $\begin{array}{l}\text { Eu uso aplicativos de mensagem instantânea } \\
\text { (Whatsapp, Messenger, etc.) }\end{array}$ & $\begin{array}{l}\text { I am able to use instant messaging software (e.g. MSN, QQ) to chat } \\
\text { with friends (Lau \& Yuen, 2014) }\end{array}$ \\
\hline & 48 & \begin{tabular}{|l|} 
Eu sei como criar documentos (doc, pdf ou \\
planilha etc.) ou apresentações de slides no \\
computador
\end{tabular} & $\begin{array}{l}\text { In this class, I construct ICT-based materials (e.g., PowerPoint slides, } \\
\text { word documents, mind maps) to represent my understanding (Chai et } \\
\text { al., 2015) }\end{array}$ \\
\hline & 49 & $\begin{array}{l}\text { Eu consigo usar aparelhos eletrônicos } \\
\text { (Computador, internet, celular, etc.) para } \\
\text { fazer trabalhos }\end{array}$ & $\begin{array}{l}\text { When an assignment/task requires the use of digital tools, I am } \\
\text { confident that I will do a great job (Siddiq et al., 2017) }\end{array}$ \\
\hline & 50 & $\begin{array}{l}\text { Eu entendo a importância de ter cuidado } \\
\text { com minhas informações pessoais na } \\
\text { internet }\end{array}$ & $\begin{array}{l}\text { Descrever os prós e contras entre permitir que uma informação seja } \\
\text { pública ou manter informações privadas e seguras (Wangenheim, } \\
\text { Alves, Weber, 2017) }\end{array}$ \\
\hline \multirow[t]{5}{*}{$\begin{array}{l}\text { Proficiência em } \\
\text { computação }\end{array}$} & 51 & $\begin{array}{l}\text { Eu consigo criar programas de computador } \\
\text { (jogos, apps, etc.) }\end{array}$ & $\begin{array}{l}\text { Develop programs for multiple computing platforms (e.g., computer } \\
\text { desktop, web, mobile, etc.) (CSTA, 2017) }\end{array}$ \\
\hline & 52 & $\begin{array}{l}\text { Eu consigo identificar as partes mais } \\
\text { importantes de um computador }\end{array}$ & $\begin{array}{l}\text { Identify the functionality of various categories of hardware } \\
\text { components and communication between them (e.g., physical layers, } \\
\text { logic gates, chips, input and output devices) (CSTA, 2017) }\end{array}$ \\
\hline & 53 & Eu sei o perigo de usar uma senha simples & $\begin{array}{l}\text { Explain the principles of information security (confidentiality, integrity } \\
\text { and availability) and authentication techniques (CSTA, 2017) }\end{array}$ \\
\hline & 54 & $\begin{array}{l}\text { Eu sei como computadores se comunicam } \\
\text { pela internet }\end{array}$ & $\begin{array}{l}\text { Describe key protocols and underlying processes of internet-based } \\
\text { services (e.g., HTTP/HTTPS and SMTP/IMAP, routing protocols) } \\
\text { (CSTA, 2017) }\end{array}$ \\
\hline & 55 & $\begin{array}{l}\text { Eu sei como identificar, testar e corrigir } \\
\text { erros de um programa de computador }\end{array}$ & I can fix an error while testing a program (Tsai, Wang \& Hsu, 2018) \\
\hline \multirow[t]{6}{*}{$\begin{array}{l}\text { Cidadania global e } \\
\text { local }\end{array}$} & 56 & Eu tenho o direito de dar minha opinião & $\begin{array}{l}\text { Knowledge of civil rights and the constitution of the home country, the } \\
\text { scope of its government (Binkley et al., 2011) }\end{array}$ \\
\hline & 57 & $\begin{array}{l}\text { Eu presto atenção nas notícias que aparecem } \\
\text { nas mídias (TV, redes sociais, sites, etc.) }\end{array}$ & $\begin{array}{l}\text { Critical reception of information from mass media (Binkley et al., } \\
\text { 2011) }\end{array}$ \\
\hline & 58 & $\begin{array}{l}\text { Eu respeito que pessoas podem ter diferentes } \\
\text { culturas, religiões, estilos de vida e opiniões }\end{array}$ & $\begin{array}{l}\text { Appreciation and understanding of differences between value systems } \\
\text { of different religious or ethnic groups (Binkley et al., 2011); Learning } \\
\text { from and working collaboratively with individuals representing diverse } \\
\text { cultures, religions and lifestyles in a spirit of mutual respect and open } \\
\text { dialogue in personal, work and community contexts (P21, 2015) }\end{array}$ \\
\hline & 59 & $\begin{array}{l}\text { Eu falo/entendo bem outro idioma (inglês, } \\
\text { espanhol, etc.) além do português }\end{array}$ & $\begin{array}{l}\text { Understanding other nations and cultures, including the use of non- } \\
\text { English languages }(\mathrm{P} 21,2015)\end{array}$ \\
\hline & 60 & $\begin{array}{l}\text { Eu consigo ter um bom relacionamento com } \\
\text { pessoas com personalidades ou interesses } \\
\text { bem diferentes dos meus }\end{array}$ & $\begin{array}{l}\text { I can hang around with classmates with personalities and interests very } \\
\text { different from mine (Kang et al., 2010) }\end{array}$ \\
\hline & 61 & $\begin{array}{l}\text { Eu sou amigável e gentil com novos colegas } \\
\text { de classe }\end{array}$ & I am usually nice to new students in the class (Kang et al., 2010) \\
\hline \multirow[t]{6}{*}{$\begin{array}{l}\text { Responsabilidade } \\
\text { social e pessoal }\end{array}$} & 62 & $\begin{array}{l}\text { Eu posso aprender muitas coisas com outras } \\
\text { pessoas }\end{array}$ & $\begin{array}{l}\text { I'm sure I have much to learn from others in terms of content } \\
\text { knowledge (mathematics, science, social studies, Norwegian) (Siddiq } \\
\text { et al., 2017) }\end{array}$ \\
\hline & 63 & Eu posso ensinar algo a outras pessoas & $\begin{array}{l}\text { I'm sure others have a lot to learn from me in terms of content } \\
\text { knowledge (mathematics, science, social studies, Norwegian) (Siddiq } \\
\text { et al., 2017) }\end{array}$ \\
\hline & 64 & $\begin{array}{l}\text { Eu me esforço o máximo possível para } \\
\text { cumprir as promessas que eu faço }\end{array}$ & $\begin{array}{l}\text { I try my best to keep promises I made with myself or with others } \\
\text { (Kang et al., 2010) }\end{array}$ \\
\hline & 65 & $\begin{array}{l}\text { Eu trato as pessoas como gostaria de ser } \\
\text { tratado(a) }\end{array}$ & Is polite to adults and peers (IFL, 2015) \\
\hline & 66 & Eu admito meus erros e peço desculpas & $\begin{array}{l}\text { When I did something dishonest, I try to rectify it (Kang et al., 2010); I } \\
\text { admit when I'm wrong (Petway et al., 2016) }\end{array}$ \\
\hline & 67 & $\begin{array}{l}\text { Eu sei que as decisões do governo podem } \\
\text { me afetar de diferentes maneiras }\end{array}$ & $\begin{array}{l}\text { Understanding the local and global implications of civic decisions } \\
\text { (P21, 2015) }\end{array}$ \\
\hline
\end{tabular}




\begin{tabular}{|c|c|c|c|}
\hline \multirow[t]{9}{*}{ Vida e carreira } & 68 & $\begin{array}{l}\text { Eu imagino onde/no que quero trabalhar } \\
\text { quando crescer }\end{array}$ & $\begin{array}{l}\text { Identify and plan for personal and professional development over time } \\
\text { and in response to change and opportunity (Binkley et al., 2011); } \\
\text { Understand models for long, medium and short-term planning and } \\
\text { balance tactical (short-term) and strategic (long-term) goals (Binkley et } \\
\text { al., 2011); I have dreams and goals that I can clearly explain to others } \\
\text { (Kang et al., 2010) }\end{array}$ \\
\hline & 69 & $\begin{array}{l}\text { Eu aceito críticas mesmo quando acredito } \\
\text { que fiz um bom trabalho }\end{array}$ & $\begin{array}{l}\text { Incorporate feedback and deal effectively with praise, setbacks and } \\
\text { criticism (Binkley et al., 2011) }\end{array}$ \\
\hline & 70 & Eu sempre faço minhas tarefas da escola & $\begin{array}{l}\text { I complete my language arts homework on time (Kyllonen, 2012); I } \\
\text { usually submit school assignments on time (Kang et al., 2010); I finish } \\
\text { my homework on time (Petway et al., 2016) }\end{array}$ \\
\hline & 71 & $\begin{array}{l}\text { Se recebo uma nota baixa na escola, tento } \\
\text { entender o porquê }\end{array}$ & $\begin{array}{l}\text { If I get lower grades than I expected, I try to find out why (Kang et al., } \\
\text { 2010) }\end{array}$ \\
\hline & 72 & Eu faço listas de coisas que tenho que fazer & $\begin{array}{l}\text { I like to make lists of things to do for school (Kyllonen, 2012); I take } \\
\text { good care of the list of things I have to do (Kang et al., 2010) }\end{array}$ \\
\hline & 73 & $\begin{array}{l}\text { Eu consigo fazer minha tarefa de casa } \\
\text { sozinho(a) }\end{array}$ & $\begin{array}{l}\text { I am a hard worker (Duckworth et al., 2007); } \\
\text { I enjoy homework (Kyllonen, 2012) }\end{array}$ \\
\hline & 74 & $\begin{array}{l}\text { Eu evito ao máximo conversar ou mexer no } \\
\text { celular durante a aula }\end{array}$ & Pays attention and resists distractions (IFL, 2015) \\
\hline & 75 & $\begin{array}{l}\text { Eu consigo me adaptar a mudanças na } \\
\text { minha rotina }\end{array}$ & $\begin{array}{l}\text { Be prepared to adapt to varied responsibilities, schedules and contexts } \\
\text { (Binkley et al., 2011) }\end{array}$ \\
\hline & 76 & $\begin{array}{l}\text { Eu consigo alcançar os objetivos que eu crio } \\
\text { para mim mesmo(a) }\end{array}$ & $\begin{array}{l}\text { Set and meet goals, prioritize, plan and manage work to achieve the } \\
\text { intended result even in the face of obstacles and competing pressures } \\
\text { (Binkley et al., 2011) }\end{array}$ \\
\hline \multirow[t]{3}{*}{$\begin{array}{l}\text { Proficiência em } \\
\text { saúde }\end{array}$} & 77 & $\begin{array}{l}\text { Eu entendo o que é necessário para ter uma } \\
\text { vida saudável }\end{array}$ & $\begin{array}{l}\text { Understanding preventive physical and mental health measures, } \\
\text { including proper diet, nutrition, exercise, risk avoidance and stress } \\
\text { reduction (P21, 2015) }\end{array}$ \\
\hline & 78 & Eu sei como prevenir a dengue & $\begin{array}{l}\text { Understanding national and international public health and safety } \\
\text { issues (P21, 2015) }\end{array}$ \\
\hline & 79 & $\begin{array}{l}\text { Eu sei como me cuidar para não ficar } \\
\text { resfriado(a) }\end{array}$ & $\begin{array}{l}\text { Establishing and monitoring personal and family health goals (P21, } \\
\text { 2015) }\end{array}$ \\
\hline \multirow[t]{3}{*}{$\begin{array}{l}\text { Consciência } \\
\text { ambiental }\end{array}$} & 80 & Eu sei as causas do aquecimento global & $\begin{array}{l}\text { Demonstrate knowledge and understanding of the environment and the } \\
\text { circumstances and conditions affecting it, particularly as relates to air, } \\
\text { climate, land, food, energy, water and ecosystems (P21, 2015) }\end{array}$ \\
\hline & 81 & Eu separo o lixo orgânico do reciclável & $\begin{array}{l}\text { Take individual and collective action towards addressing } \\
\text { environmental challenges (e.g., participating in global actions, } \\
\text { designing solutions that inspire action on environmental issues) (P21, } \\
\text { 2015) }\end{array}$ \\
\hline & 82 & $\begin{array}{l}\text { Eu tento não demorar no banho para } \\
\text { economizar água }\end{array}$ & $\begin{array}{l}\text { Take individual and collective action towards addressing } \\
\text { environmental challenges (e.g., participating in global actions, } \\
\text { designing solutions that inspire action on environmental issues) (P21, } \\
\text { 2015) }\end{array}$ \\
\hline
\end{tabular}

\section{Avaliação do Modelo bASE21}

Com o objetivo de avaliar a confiabilidade e a validade do instrumento de medição do modelo bASE21, o mesmo foi aplicado em um estudo de caso no contexto da educação básica.

\subsection{Definição da Avaliação}

O objetivo da avaliação do modelo é avaliar a confiabilidade e validade do questionário de autoavaliação desenvolvido como instrumento de medição do modelo. Para isso são analisadas as seguintes perguntas:

PA1: Há evidências de consistência interna no instrumento de medição?

PA2: Há evidências de validade convergente e discriminante no instrumento de medição? 
Os dados para a análise são coletados a partir da aplicação do questionário em um estudo de caso one-shot na educação básica (sem aplicação de nenhum tratamento específico). Os dados são coletados por meio do preenchimento do questionário, disponibilizado de maneira impressa ou online, por estudantes de diferentes turmas da educação básica.

\subsection{Execução da Avaliação}

O questionário foi aplicado durante os meses de abril e maio de 2018. Participaram da pesquisa um total de 159 estudantes da educação básica com idades de 8 a 19 anos (Figura 4) nos estados de Santa Catarina e Rio Grande do Sul/Brasil.

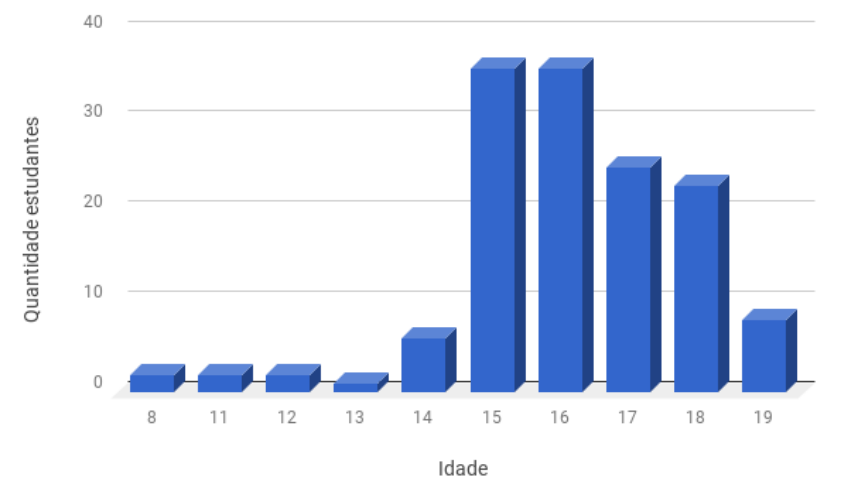

Figura 4: Distribuição da idade dos estudantes que participaram da avaliação.

\subsection{Análise dos Dados}

Os dados coletados foram agrupados em uma única amostra, de modo a obter uma maior precisão e poder estatístico. Antes da análise estatística, também revisou-se a completude dos dados coletados. Foram excluídos os dados de questionários deixados em branco ou que tiveram poucos itens respondidos (menos de 10 itens respondidos). Como resultado, foram excluídos 11 questionários respondidos, deixando um total de 148 respostas consideradas válidas.

\section{PA1: Há evidências de consistência interna no instrumento de medição?}

O questionário em sua totalidade apresenta o coeficiente alfa de Cronbach (Cronbach, 1951) de 0,958, sendo classificada como excelente consistência interna (Devellis, 2016).

Foi também realizado o cálculo do coeficiente retirando cada item, caso o mesmo fosse excluído (Tabela 6). Essa análise é realizada para identificar os possíveis itens que estão prejudicando a consistência interna do instrumento. Geralmente são removidos os itens cujo alfa de Cronbach se excluído é superior ao alfa de Cronbach do instrumento. Isso indica que se o item for excluído o alfa de Cronbach do instrumento aumenta, melhorando a consistência interna do mesmo. 
Tabela 6: Coeficiente alfa de Cronbach para cada item caso fosse excluído.

\begin{tabular}{|lc|lc|lc|lc|}
\hline Item & $\begin{array}{c}\text { Alfa se item } \\
\text { excluído }\end{array}$ & Item & $\begin{array}{c}\text { Alfa se item } \\
\text { excluído }\end{array}$ & Item & $\begin{array}{c}\text { Alfa se item } \\
\text { excluído }\end{array}$ & Item & $\begin{array}{c}\text { Alfa se item } \\
\text { excluído }\end{array}$ \\
\hline IT1 &, 958 & IT21 &, 958 & IT41 &, 958 & IT61 &, 957 \\
IT2 &, 958 & IT22 &, 958 & IT42 &, 958 & IT62 &, 957 \\
IT3 &, 958 & IT23 &, 958 & IT43 &, 958 & IT63 &, 957 \\
IT4 &, 958 & IT24 &, 958 & IT44 &, 958 & IT64 &, 957 \\
IT5 &, 958 & IT25 &, 959 & IT45 &, 958 & IT65 &, 957 \\
IT6 &, 958 & IT26 &, 958 & IT46 &, 958 & IT66 &, 958 \\
IT7 &, 957 & IT27 &, 958 & IT47 &, 958 & IT67 &, 958 \\
IT9 &, 958 & IT28 &, 958 & IT48 &, 958 & IT68 &, 958 \\
IT8 &, 958 & IT29 &, 958 & IT49 &, 958 & IT69 &, 958 \\
IT10 &, 958 & IT30 &, 958 & IT50 &, 957 & IT70 &, 958 \\
IT11 &, 958 & IT31 &, 957 & IT51 &, 958 & IT71 &, 958 \\
IT12 &, 957 & IT32 &, 957 & IT52 &, 958 & IT72 &, 958 \\
IT13 &, 958 & IT33 &, 958 & IT53 &, 958 & IT73 &, 958 \\
IT14 &, 958 & IT34 &, 958 & IT54 &, 958 & IT74 &, 958 \\
IT15 &, 958 & IT35 &, 957 & IT55 &, 958 & IT75 &, 958 \\
IT16 &, 958 & IT36 &, 958 & IT56 &, 958 & IT76 &, 958 \\
IT17 &, 958 & IT37 &, 958 & IT57 &, 958 & IT77 &, 958 \\
IT18 &, 958 & IT38 &, 958 & IT58 &, 958 & IT78 &, 958 \\
IT19 &, 958 & IT39 &, 958 & IT59 &, 959 & IT79 &, 958 \\
IT20 &, 958 & IT40 &, 957 & IT60 &, 958 & IT80 &, 957 \\
& & & & & & IT81 &, 958 \\
& & & & & & IT82 &, 958 \\
\hline
\end{tabular}

Os resultados demonstraram que dois itens (IT59: Eu falo/entendo bem outro idioma (inglês, espanhol, etc.) e IT25: Não tenho vergonha de falar em público) podem estar prejudicando levemente a consistência interna do questionário. Porém, por ser uma variação muito pequena (variação de 0.001 no alfa Cronbach) ainda não se recomenda a exclusão desses itens. Logo, nenhum dos itens parece estar prejudicando significativamente a consistência interna do questionário.

\section{PA2: Há evidências de validade convergente e discriminante nos itens do instrumento de medição?}

Para a análise da validade de constructo dos itens do questionário foi calculada a matriz de correlação policórica, indicada para variáveis qualitativas ordinais (Drasgow, 1986). Para a análise dos coeficientes foi adotado o coeficiente de Cohen. Dessa maneira as correlações moderadas são aquelas em que o coeficiente é superior a 0.29 (destacadas em verde). As correlações altas são aquelas cujo coeficiente é superior a 0.5 (destacadas em amarelo) (Cohen, 1988). Por outro lado, os coeficientes negativos (destacados em vermelho), indicam uma baixa correlação.

Quando feita a análise da correlação policórica de todos os itens do questionário, observouse que os itens das habilidades que apresentaram correlações moderadas, também apresentaram muitas correlações moderadas com os outros itens do questionário. O mesmo se repete para os itens das habilidades que apresentaram poucas correlações moderadas/altas, que apresentam poucas correlações moderadas/altas com os outros itens do questionário.

No geral, o questionário apresentou muitos itens correlacionados entre si, independente de habilidade ou grupo de habilidade. Isso indica que o modelo não identifica claramente a divisão de habilidades pelos itens, consequentemente indica que o questionário pode precisar de uma melhor divisão ou redistribuição de itens entre as habilidades. Foi também identificado um item (IT59: Eu falo/entendo bem outro idioma (inglês, espanhol, etc.) além do português) com pouca correlação com os demais itens do questionário. 
Analisando cada habilidade de maneira individual, a habilidade "Criatividade e inovação" (Tabela 7) apresentou baixa validade interna, em que a maioria dos itens não apresentou correlação entre si. Inclusive o par entre os itens IT1: Eu invento/imagino muitas coisas que ainda não existem e IT6: Eu aprendo com os meus erros ou quando minhas ideias dão errado apresentou correlação divergente, ou seja, esses itens estão aparentemente medindo fatores diferentes.

\begin{tabular}{|c|c|c|c|c|c|c|c|}
\hline \multicolumn{8}{|c|}{ Criatividade e inovação } \\
\hline & IT1 & IT2 & IT3 & IT4 & IT5 & IT6 & IT7 \\
\hline IT1 & 1 & & & & & & \\
\hline IT2 & 0,214 & 1 & & & & & \\
\hline IT3 & 0,361 & 0,334 & 1 & & & & \\
\hline IT4 & 0,198 & 0,307 & 0,197 & 1 & & & \\
\hline IT5 & 0,141 & 0,313 & 0,246 & 0,126 & 1 & & \\
\hline IT6 & $-0,046$ & 0,203 & 0,26 & 0,141 & 0,202 & 1 & \\
\hline IT7 & 0,136 & 0,199 & 0,337 & 0,298 & 0,228 & 0,452 & 1 \\
\hline
\end{tabular}

Em geral, a habilidade "Pensamento crítico, resolução de problemas e tomada de decisão" (Tabela 8) apresentou correlação moderada entre seus itens. O item IT14: Eu me pergunto se estou fazendo bem as minhas tarefas da escola apresentou uma correlação alta com o item IT13: Eu escolho e organizo o material que preciso quando vou fazer algo (tarefas de casa, trabalhos, estudar, etc.). Porém, o IT14 apresentou baixa correlação com o item IT16: Eu consigo explicar as minhas opiniões e decisões além do último também não apresentar correlação significativa com nenhum outro item da habilidade. Isso indica que o IT16 não está medindo o mesmo que os outros itens.

Tabela 8: Coeficientes de correlação de Pensamento crítico, resolução de problemas e tomada de decisão.

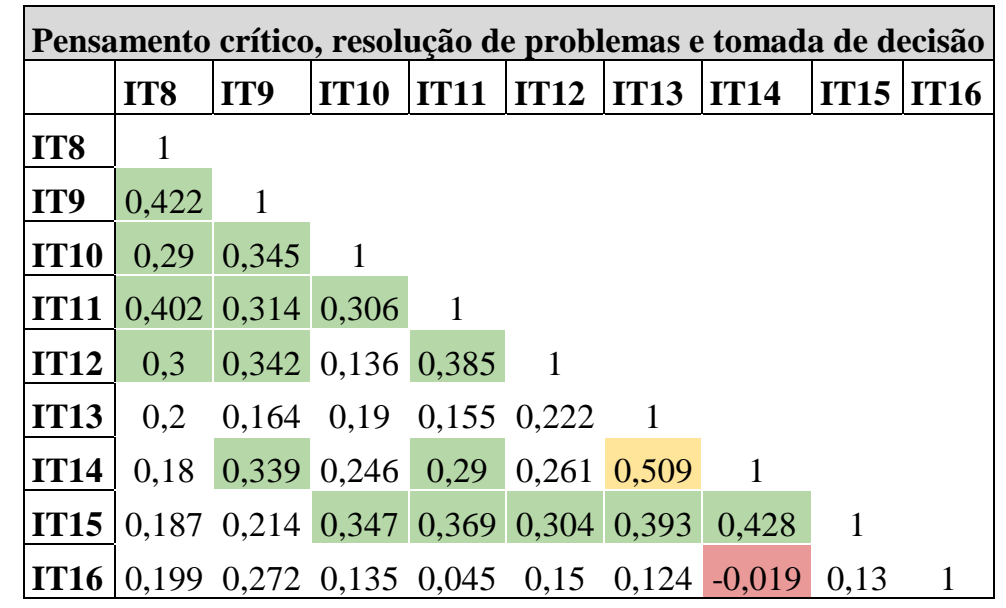

A habilidade "Aprender a aprender e metacognição" (Tabela 9) apresentou algumas correlações moderadas entre seus itens e também algumas correlações não significativas. Isso indica que não foi possível identificar com clareza se os itens da habilidade estão de fato medindo o mesmo fator. Observa-se também que o item IT17: Eu planejo como vou estudar (quais exercícios vou fazer em que dias/tempo, etc.) apresentou alta correlação com o item IT18: Se estou tendo dificuldade em um assunto da matéria eu dedico mais tempo de estudo para esse assunto indicando que esses dois itens estão medindo o mesmo fator. 
Tabela 9: Coeficientes de correlação de Aprender a aprender e metacognição.

\begin{tabular}{|c|c|c|c|c|c|}
\hline \multicolumn{7}{|c|}{ Aprender a aprender e metacognição } \\
\hline & IT17 & IT18 & IT19 & IT20 & IT21 \\
\hline IT17 & 1 & & & & \\
IT18 & 0,579 & 1 & & & \\
IT19 & 0,072 & 0,271 & 1 & & \\
IT20 & 0,209 & 0,386 & 0,366 & 1 & \\
\hline IT21 & 0,411 & 0,322 & 0,25 & 0,156 & 1 \\
\hline
\end{tabular}

Os itens da habilidade "Comunicação" (Tabela 10) apresentaram baixa correlação entre si. Também apresentou um par com correlação divergente, entre os itens IT25: Não tenho vergonha de falar em público e IT22: Eu ouço com atenção para entender o que os outros falam. $\mathrm{O}$ único item que tem correlação moderada com os outros itens é o IT27: Eu consigo fazer bons argumentos em um debate.

Tabela 10: Coeficientes de correlação de Comunicação.

\begin{tabular}{|c|c|c|c|c|c|c|}
\hline \multicolumn{7}{|c|}{ Comunicação } \\
\hline & IT22 & IT23 & IT24 & IT25 & IT26 & IT27 \\
\hline IT22 & 1 & & & & & \\
\hline IT23 & 0,277 & 1 & & & & \\
\hline IT24 & 0,223 & 0,35 & 1 & & & \\
\hline IT25 & $-0,014$ & 0,254 & 0,239 & 1 & & \\
\hline IT26 & 0,191 & 0,213 & 0,203 & 0,183 & 1 & \\
\hline IT27 & 0,236 & 0,331 & 0,426 & 0,479 & 0,344 & 1 \\
\hline
\end{tabular}

A habilidade "Colaboração e trabalho em equipe" apresentou, em geral, correlação moderada entre os seus itens (Tabela 11). É possível identificar um item (IT28: Eu gosto de trabalhar junto com os meus colegas para fazer trabalhos ou resolver problemas) que não demonstra correlação com a maioria dos outros itens, e inclusive apresenta uma baixa correlação com o item IT30: Eu gosto de ser o(a) líder do grupo. O item IT29: Eu consigo arranjar um tempo para ajudar outras pessoas, apresentou maior correlação com os itens da habilidade "Responsabilidade Social e Pessoal" (Tabela 12), o que indica que ele está na realidade medindo a habilidade "Responsabilidade social e pessoal" e deveria ser reagrupado. 
Tabela 11: Coeficientes de correlação da habilidade Colaboração e trabalho em equipe.

\begin{tabular}{|c|c|c|c|c|c|c|c|c|c|c|c|}
\hline \multicolumn{12}{|c|}{ Colaboração e trabalho em equipe } \\
\hline & IT28 & IT29 & IT30 & IT31 & IT32 & IT33 & IT34 & IT35 & IT36 & IT37 & IT38 \\
\hline IT28 & 1 & & & & & & & & & & \\
\hline IT29 & 0,163 & 1 & & & & & & & & & \\
\hline IT30 & $-0,22$ & 0,247 & 1 & & & & & & & & \\
\hline IT31 & 0,133 & 0,264 & 0,303 & 1 & & & & & & & \\
\hline IT32 & 0,279 & 0,322 & 0,287 & 0,44 & 1 & & & & & & \\
\hline IT33 & 0,285 & 0,349 & 0,15 & 0,345 & 0,407 & 1 & & & & & \\
\hline IT34 & 0,22 & 0,301 & 0,095 & 0,41 & 0,277 & 0,474 & 1 & & & & \\
\hline IT35 & 0,244 & 0,248 & 0,265 & 0,6 & 0,637 & 0,552 & 0,436 & 1 & & & \\
\hline IT36 & 0,149 & 0,264 & 0,292 & 0,333 & 0,334 & 0,419 & 0,175 & 0,376 & 1 & & \\
\hline IT37 & 0,251 & 0,201 & 0,296 & 0,388 & 0,365 & 0,224 & 0,336 & 0,31 & 0,19 & 1 & \\
\hline IT38 & 0,305 & 0,314 & 0,173 & 0,365 & 0,443 & 0,283 & 0,292 & 0,346 & 0,31 & 0,573 & 1. \\
\hline
\end{tabular}

Tabela 12: Comparação da correlação do IT29 com os itens da habilidade Responsabilidade social e pessoal.

\begin{tabular}{|l|l|l|l|l|l|l|}
\hline \multicolumn{6}{|c|}{ Responsabilidade social e pessoal } \\
\hline & IT62 & IT63 & IT64 & IT65 & IT66 & IT67 \\
\hline IT29 & 0,297 & 0,424 & 0,401 & 0,436 & 0,399 & 0,197 \\
\hline
\end{tabular}

A análise da correlação dos itens referentes a habilidade "Proficiência em informação" (Tabela 13) apresentou bons resultados em sua maioria. Dentro dos itens da habilidade, um par apresentou alta correlação (IT41: Eu posso mudar de opinião dependendo de quanto eu sei sobre o assunto e IT42: Eu consigo explicar porque mudei de opinião). Porém, também se observou que o item IT45: Quando eu estudo, eu busco mais informações além das anotações do meu caderno ou apostila/livro apresentou baixa correlação com os outros itens da habilidade. Uma causa desse resultado pode ter ocorrido por causa da formulação do item, sendo que aparentemente ele trata de um assunto relevante à habilidade, a capacidade de buscar informações além do básico.

\begin{tabular}{|c|c|c|c|c|c|c|c|}
\hline \multicolumn{8}{|c|}{ Proficiência em informação } \\
\hline & IT39 & IT40 & IT41 & IT42 & IT43 & IT44 & IT45 \\
\hline IT39 & 1 & & & & & & \\
\hline IT40 & 0,488 & 1 & & & & & \\
\hline IT41 & 0,357 & 0,394 & 1 & & & & \\
\hline IT42 & 0,412 & 0,42 & 0,561 & 1 & & & \\
\hline IT43 & 0,35 & 0,364 & 0,36 & 0,463 & 1 & & \\
\hline IT44 & 0,299 & 0,337 & 0,409 & 0,299 & 0,297 & 1 & \\
\hline IT45 & 0,444 & 0,27 & 0,235 & 0,147 & 0,195 & 0,332 & 1 \\
\hline
\end{tabular}

A análise dos itens referente a habilidade "Proficiência em TIC" (Tabela 14) demonstrou bons resultados em todos os pares de itens, com a maioria indicando alta correlação entre eles. Observou-se somente a exceção de três pares, que ainda assim indicam uma correlação moderada. A princípio não é necessária nenhuma mudança nos itens dessa habilidade. 
Tabela 14: Coeficientes de correlação de Proficiência em TIC.

\begin{tabular}{|c|c|c|c|c|c|}
\hline \multicolumn{6}{|c|}{ Proficiência em TIC } \\
\hline & IT46 & IT47 & IT48 & IT49 & IT50 \\
\hline IT46 & 1 & & & & \\
\hline IT47 & 0,39 & 1 & & & \\
\hline IT48 & 0,526 & 0,613 & 1 & & \\
\hline IT49 & 0,571 & 0,618 & 0,782 & 1 & \\
\hline IT50 & 0,474 & 0,483 & 0,58 & 0,684 & 1 \\
\hline
\end{tabular}

A análise dos itens referente a habilidade "Proficiência em computação" (Tabela 15) também apresentou bons resultados de validade interna quando avaliada a correlação dos seus itens internos. Apenas dois pares não apresentaram correlação, entre ele os itens IT51: Eu consigo criar programas de computador (jogos, apps, etc.) e IT53: Eu sei o perigo de usar uma senha simples) apresentaram uma baixa correlação, com um coeficiente muito próximo a zero. Porém, como esse par de itens apresenta correlações moderadas com o resto dos itens, é necessária uma melhor investigação do motivo dessa baixa correlação entre os itens.

\begin{tabular}{|c|c|c|c|c|c|}
\hline \multicolumn{6}{|c|}{ Proficiência em computação } \\
\hline & IT51 & IT52 & IT53 & IT54 & IT55 \\
\hline IT51 & 1 & & & & \\
\hline IT52 & 0,332 & 1 & & & \\
\hline IT53 & 0,068 & 0,44 & 1 & & \\
\hline IT54 & 0,412 & 0,658 & 0,443 & 1 & \\
\hline IT55 & 0,649 & 0,428 & 0,233 & 0,468 & 1 \\
\hline
\end{tabular}

A análise dos itens referente a habilidade "Cidadania global e local" (Tabela 16) apresentou correlação moderada entre a maioria de seus itens com exceção do item IT59: Eu falo/entendo bem outro idioma (inglês, espanhol, etc.) além do português. Além de não apresentar correlação com os itens de sua habilidade, o item IT59 não apresentou correlação com nenhum item do questionário, indicando que ele não está medindo nenhuma das habilidades definidas nesse trabalho, e deve ser removido do questionário.

\begin{tabular}{|c|c|c|c|c|c|c|}
\hline \multicolumn{7}{|c|}{ Cidadania global e local } \\
\hline & IT56 & IT57 & IT58 & IT59 & IT60 & IT61 \\
\hline IT56 & 1 & & & & & \\
\hline IT57 & 0,151 & 1 & & & & \\
\hline IT58 & 0,536 & 0,209 & 1 & & & \\
\hline IT59 & 0,181 & 0,086 & 0,13 & 1 & & \\
\hline IT60 & 0,323 & 0,37 & 0,506 & 0,129 & 1 & \\
\hline IT61 & 0,514 & 0,243 & 0,417 & 0,071 & 0,528 & 1 \\
\hline
\end{tabular}

Os itens da habilidade "Responsabilidade social e pessoal" (Tabela 17) apresentaram, no geral, correlação moderada entre si. Um par (IT64: Eu me esforço o máximo possível para cumprir as promessas que eu faço e IT67: Eu sei que as decisões do governo podem me afetar de diferentes maneiras) não apresentou correlação significativa. 
Tabela 17: Coeficientes de correlação da habilidade Responsabilidade social e pessoal.

\begin{tabular}{|c|c|c|c|c|c|c|}
\hline \multicolumn{7}{|c|}{ Responsabilidade social e pessoal } \\
\hline & IT62 & IT63 & IT64 & IT65 & IT66 & IT67 \\
\hline IT62 & 1 & & & & & \\
\hline IT63 & 0,682 & & & & & \\
\hline IT64 & 0,476 & 0,465 & 1 & & & \\
\hline IT65 & 0,553 & 0,514 & 0,471 & 1 & & \\
\hline IT66 & 0,32 & 0,355 & 0,425 & 0,499 & 1 & \\
\hline IT67 & 0,538 & 0,482 & 0,194 & 0,311 & 0,335 & 1 \\
\hline
\end{tabular}

A análise da correlação da habilidade "Vida e carreira" (Tabela 18) apresentou poucas correlações entre seus itens, indicando uma baixa validade interna dos seus itens. O IT69: Eu aceito críticas mesmo quando acredito que fiz um bom trabalho, que apresentou pouca correlação com os outros itens da habilidade, por outro lado, apresentou correlação moderada/alta com os itens da habilidade "Responsabilidade social e pessoal" (Tabela 19), o que indica que ele pode estar inserido na habilidade errada.

Tabela 18: Coeficientes de correlação da habilidade Vida e carreira.

\begin{tabular}{|c|c|c|c|c|c|c|c|c|c|}
\hline \multicolumn{10}{|c|}{ Vida e carreira } \\
\hline & IT68 & IT69 & IT70 & IT71 & IT72 & IT73 & IT74 & IT75 & IT76 \\
\hline IT68 & 1 & & & & & & & & \\
\hline IT69 & 0,472 & 1 & & & & & & & \\
\hline IT70 & 0,216 & 0,163 & 1 & & & & & & \\
\hline IT71 & 0,306 & 0,245 & 0,409 & 1 & & & & & \\
\hline IT72 & 0,308 & 0,178 & 0,474 & 0,295 & 1 & & & & \\
\hline IT73 & 0,388 & 0,201 & 0,253 & 0,373 & 0,199 & 1 & & & \\
\hline IT74 & 0,123 & 0,145 & 0,371 & 0,313 & 0,337 & 0,211 & 1 & & \\
\hline IT75 & 0,364 & 0,291 & 0,116 & 0,37 & 0,19 & 0,346 & 0,203 & 1 & \\
\hline IT76 & 0,225 & 0,332 & 0,28 & 0,337 & 0,251 & 0,229 & 0,176 & 0,39 & 1 \\
\hline
\end{tabular}

Tabela 19: Comparação da correlação do IT69 com os itens da habilidade Responsabilidade social e pessoal.

\begin{tabular}{|l|l|l|l|l|l|l|}
\hline \multicolumn{7}{|c|}{ Responsabilidade social e pessoal } \\
\hline & IT62 & IT63 & IT64 & IT65 & IT66 & IT67 \\
\hline IT69 & 0,301 & 0,377 & 0,316 & 0,522 & 0,529 & 0,301 \\
\hline
\end{tabular}

Uma habilidade que apresentou ótimos resultados quanto a sua validade interna foi a habilidade "Proficiência em saúde" (Tabela 20). Todos os itens da habilidade apresentaram alta correlação entre si, indicando que todos estão medindo o mesmo fator.

Tabela 20: Coeficientes de correlação de Proficiência em saúde.

\begin{tabular}{|c|c|c|c|}
\hline \multicolumn{4}{|c|}{ Proficiência em saúde } \\
\hline & IT77 & IT78 & IT79 \\
\hline IT77 & 1 & & \\
\hline IT78 & 0,558 & 1 & \\
\hline IT79 & 0,574 & 0,709 & 1 \\
\hline
\end{tabular}


A habilidade "Consciência ambiental" apresentou somente um par (IT81: Eu separo o lixo orgânico do reciclável e IT82: Eu tento não demorar no banho para economizar água) com correlação moderada (Tabela 21), indicando que o item IT80: Eu sei as causas do aquecimento global mede outro fator. Isso é confirmado quando o IT80 é analisado com os itens da habilidade "Proficiência em saúde" (Tabela 22), apresentando alta correlação com todos os itens da habilidade, o que indica que o IT80 deve ser reagrupado na habilidade "Proficiência em saúde".

Tabela 21: Coeficientes de correlação da habilidade Consciência ambiental.

\begin{tabular}{|c|c|c|c|}
\hline \multicolumn{5}{|c|}{ Consciência ambiental } \\
\hline & IT80 & IT81 & IT82 \\
\hline IT80 & 1 & & \\
\cline { 1 - 1 } IT81 & 0,258 & 1 & \\
\cline { 1 - 2 } IT82 & 0,179 & 0,336 & 1 \\
\hline
\end{tabular}

Tabela 22: Comparação da correlação do IT80 com os itens da habilidade Proficiência em saúde.

\begin{tabular}{|c|c|c|c|}
\hline \multicolumn{4}{|c|}{ Proficiência em saúde } \\
\hline & IT77 & IT78 & IT79 \\
\hline IT80 & 0,536 & 0,691 & 0,685 \\
\hline
\end{tabular}

Em geral, a análise da maioria das habilidades apresentou resultados promissores quanto a sua validade interna. Observou-se alguns itens que parecem ser associadas de forma incorreta a habilidades, apresentando maior correlação com itens de habilidade diferentes da que eles foram associados.

Além da análise da correlação dos itens internos de cada habilidade, também foi analisada a correlação dos itens entre habilidades, com o objetivo de investigar se habilidades diferentes estão medindo fatores diferentes e a existência de grupos de habilidades relacionadas. No geral, as habilidades apresentaram pouca correlação entre si, com exceção da habilidade "Responsabilidade social e pessoal" que apresentou correlações moderadas com as habilidades "Proficiência em informação", "Proficiência em TIC", "Proficiência em saúde" e "Colaboração e trabalho em equipe". Além disso, observou-se que os itens da habilidade "Responsabilidade social e pessoal" tem correlação moderada com boa parte dos itens do questionário. Isso pode indicar que a habilidade está muito vaga, ou que ela se relaciona com muitas das outras habilidades. É necessária uma melhor investigação de porque essa habilidade apresentou esse resultado.

Quanto a correlação discriminante, poucas habilidades apresentaram resultados conclusivos. As habilidades "Proficiência em computação" e "Aprender a aprender e metacognição" aparentam estar medindo fatores diferentes de fato (Tabela 23), mas ainda assim existe uma correlação entre seus itens (IT53: Eu sei o perigo de usar uma senha simples e IT20: Eu gosto de aprender coisas novas), apesar de semanticamente tratarem de assuntos bem diferentes. 
Tabela 23: Correlação entre Proficiência em computação e Aprender a aprender e metacognição.

\begin{tabular}{|l|c|c|c|c|c|c|}
\hline \multicolumn{2}{|c|}{} & \multicolumn{5}{|c|}{ Aprender a aprender e metacognição } \\
\cline { 3 - 8 } \multicolumn{1}{|c|}{} & IT17 & IT18 & IT19 & IT20 & IT21 \\
\hline \multirow{4}{*}{ Proficiência em computação } & IT51 & 0,088 & 0,103 & 0,279 & 0,095 & 0,137 \\
\cline { 2 - 8 } & IT52 & $-0,058$ & 0,031 & 0,28 & 0,218 & 0,163 \\
\cline { 2 - 8 } & IT53 & 0,127 & 0,27 & 0,269 & 0,306 & $-0,015$ \\
\cline { 2 - 7 } & IT54 & 0,088 & 0,056 & 0,158 & 0,161 & 0,051 \\
\cline { 2 - 7 } & IT55 & $-0,001$ & $-0,059$ & 0,236 & 0,012 & 0,132 \\
\hline
\end{tabular}

\subsection{Proposta de Modificações}

A partir da análise dos dados é possível identificar algumas oportunidades de melhoria do modelo bASES21 apresentadas na Tabela 24.

Tabela 24: Sugestões de modificações no modelo bASES21.

\begin{tabular}{|l|l|l|}
\hline \multicolumn{1}{|c|}{ Item } & \multicolumn{1}{|c|}{ Análise } & \multicolumn{1}{|c|}{ Sugestão } \\
\hline $\begin{array}{l}\text { IT6: Eu aprendo com os meus } \\
\text { erros ou quando minhas ideias dão } \\
\text { errado (Criatividade e inovação) }\end{array}$ & $\begin{array}{l}\text { Pouco correlacionado com os itens da sua } \\
\text { habilidade. Está moderadamente correlacionado } \\
\text { com a maioria dos itens de "Pensamento crítico, } \\
\text { resolução de problemas e tomada de decisão" }\end{array}$ & $\begin{array}{l}\text { Reagrupar à Pensamento } \\
\text { crítico, resolução de } \\
\text { problemas e tomada de } \\
\text { decisão }\end{array}$ \\
\hline $\begin{array}{l}\text { IT16: Eu consigo explicar as } \\
\text { minhas opiniões e decisões } \\
\text { (Pensamento crítico, resolução de } \\
\text { problemas e tomada de decisão) }\end{array}$ & $\begin{array}{l}\text { Tem correlação moderada com a maioria dos } \\
\text { itens de "Comunicação" }\end{array}$ & Reagrupar à Comunicação \\
\hline $\begin{array}{l}\text { IT22: Eu ouço com atenção para } \\
\text { entender o que os outros falam } \\
\text { (Comunicação) }\end{array}$ & $\begin{array}{l}\text { Não possui correlação com os itens da sua } \\
\text { habilidade. Possui correlação moderada com a } \\
\text { maioria dos itens de "Colaboração e trabalho em } \\
\text { equipe" e "Responsabilidade social e pessoal" }\end{array}$ & $\begin{array}{l}\text { Reagrupar à Colaboração e } \\
\text { trabalho em equipe }\end{array}$ \\
\hline $\begin{array}{l}\text { IT34: Eu respeito as diferenças } \\
\text { das pessoas de outras regióes, } \\
\text { países e religiões (Colaboração e } \\
\text { trabalho em equipe) }\end{array}$ & $\begin{array}{l}\text { Item com formulação, significado e correlações } \\
\text { iguais ao IT58: Eu respeito que pessoas podem } \\
\text { ter diferentes culturas, religiões, estilos de vida e } \\
\text { opiniões }\end{array}$ & $\begin{array}{l}\text { Remover (item duplicado ao } \\
\text { IT58) }\end{array}$ \\
\hline $\begin{array}{l}\text { IT59: Eu falo/entendo bem outro } \\
\text { idioma (inglês, espanhol, etc. } \\
\text { além do português (Cidadania } \\
\text { global e local) }\end{array}$ & $\begin{array}{l}\text { Se correlaciona só com o item IT42: Eu consigo } \\
\text { explicar porque mudei de opinião em todo } \\
\text { questionário. }\end{array}$ & $\begin{array}{l}\text { Remover (nenhuma } \\
\text { correlação com os outros } \\
\text { itens) }\end{array}$ \\
\hline $\begin{array}{l}\text { IT69: Eu aceito criticas mesmo } \\
\text { quando acredito que fiz um bom } \\
\text { trabalho (Vida e carreira) }\end{array}$ & $\begin{array}{l}\text { Tem pouca correlação com os outros itens da sua } \\
\text { habilidade. Se correlaciona com todos os itens de } \\
\text { "Responsabilidade social e pessoal" }\end{array}$ & $\begin{array}{l}\text { Reagrupar à } \\
\text { Responsabilidade social e } \\
\text { pessoal }\end{array}$ \\
\hline
\end{tabular}

\subsection{Ameaças à Validade}

Levando em consideração as características da pesquisa realizada, a mesma está sujeita a ameaças à sua validade. A partir da identificação das possíveis ameaças, foram planejadas estratégias de mitigação com o objetivo de minimizar o impacto dessas ameaças nos resultados da pesquisa. Para minimizar as ameaças relacionadas ao tipo de pesquisa adotado, todos os passos para a sua realização foram sistematicamente desenvolvidos e documentados com base na literatura existente levantada de forma sistemática. Também foi realizada a revisão dos itens do instrumento por um painel de especialistas e representantes do público alvo com o objetivo de assegurar o entendimento, completude e consistência dos itens. 
A avaliação do instrumento foi sistematicamente definida e documentada afim de mitigar possíveis ameaças quanto a confiabilidade do estudo. Quanto a escolha dos métodos estatísticos para a avaliação, com o objetivo de minimizar os impactos causados pela escolha de um método de avaliação não adequado, a análise estatística dos dados seguiu as recomendações de Trochim e Donnelly (2008).

Quanto as ameaças referentes à validade externa e o tamanho da amostra, para conseguir o maior número de participantes foi disponibilizada além da versão impressa, uma versão online do questionário, facilitando a aplicação, e tornando possível a coleta de aproximadamente 160 respostas da maioria de estudantes do ensino médio. Porém, como a amostra ainda foi pequena e não igualmente distribuída nos níveis educacionais, recomenda-se a revisão dos resultados em novos estudos com amostras maiores, permitindo também a aplicação de outras análises estatísticas, como a análise fatorial, visando uma investigação mais detalhada dos fatores medidos. Mesmo assim, essa avaliação inicial demonstrou potencial quanto a sua confiabilidade, e a validade, das definições e agrupamentos, das habilidades do século XXI.

\section{Conclusão}

Este artigo apresenta o desenvolvimento de um modelo de avaliação de habilidades do século XXI no contexto do ensino da computação por meio de unidades instrucionais na educação básica. Com base nos resultados de um mapeamento sistemático da literatura, o modelo bASES21 é desenvolvido de forma sistemática incluindo como instrumento de medição, um questionário de autoavaliação.

A avaliação do modelo bASES21, com base em um total de 148 respostas, indicou alta confiabilidade interna (alfa de Cronbach $=0,958$ ), porém apresentou resultados inconclusivos quanto a sua validade, indicando habilidades com alta correlação interna dos seus itens, o que mostra que todos estão medindo um mesmo fator/habilidade, e também indicando outras habilidades cujos seus itens não se correlacionam entre si, indicando que estão medindo outros fatores/habilidades. Porém, foram identificados alguns itens que não apresentam muita correlação com os outros itens do questionário, assim como alguns itens que estão correlacionados com vários outros itens, de diferentes habilidades, indicando que talvez sua formulação foi muito abrangente e não específica para uma habilidade. Com base nesses resultados da avaliação foi refinado a versão 1.0 do modelo bASES21, porém se prevê a realização de estudos com amostras maiores detalhando a análise da estrutura do modelo.

Diferente aos modelos já existentes, o modelo bASE21 contempla todas as habilidades definidas pelos principais frameworks referentes ao ensino de habilidades do século XXI ATC21 (Binkley et al., 2011) e P21 (2015) acrescentando também a habilidade de proficiência em computação. O modelo bASE21 representa um passo inicial na pesquisa de modelos de avaliação de habilidades do século XXI adotando o método de autoavaliação. Neste contexto, também se planeja como trabalho futuro a inclusão de outras formas de coleta de dados (p.ex. por testes, entrevistas) viabilizando uma triangulação dos dados coletados visando o aumento da precisão dos resultados obtidos.

\section{Agradecimentos}

Gostaríamos agradecer a todos que ajudaram na coleta de dados.

Este trabalho foi financiado pelo CNPq (Conselho Nacional de Desenvolvimento Científico e Tecnológico), uma entidade do governo brasileiro focado no desenvolvimento científico e tecnológico e pelo Programa da Iniciação à Pesquisa Institucional (PIBIC/UFSC). 


\section{Referências}

ACM (Association for Computing Machinery). (2005). Computing Curricula 2005. Disponível em: https://www.acm.org/education/curricula-recommendations

Aesaert, K., Nijlen, D. V., Vanderlinde, R. \& Braak, J. V. (2014). Direct measures of digital information processing and communication skills in primary education: Using item response theory for the development and validation of an ICT competence scale. Computers \& Education, 76, p. 168-181. doi: 10.1016/j.compedu.2014.03.013. [GS Search].

Anderson, R. (2008). Implications of the information and knowledge society for education. In J. Voogt \& G. Knezek (Eds.), International Handbook of Information Technology in Primary and Secondary Education. New York: Springer. doi: 10.1007/978-0-387-73315-9 1. [GS Search].

Base Nacional Comum Curricular (BNCC). Disponível em: http://basenacionalcomum.mec.gov.br/

Basili, V. R.; Caldeira, G.; Rombach, H. D. (1994). Goal Question Metric Paradigm. In: Encyclopedia of Software Engineering, John Wiley \& Sons.

Beecham, S., Hall, T., Britton, C., Cottee, M., \& Rainer, A. (2005). Using an Expert Panel to Validate a Requirements Process Improvement Model. Journal of Systems and Software, 76(3), 251-275. doi: 10.1016/j.jss.2004.06.004. [GS Search].

Binkley, M., Ola, E., Herman, J. Raizen, S., Ripley, M. \& Rumble, M. (2011). Defining 21st Century Skills. In: Assessment and teaching of $21^{\text {st }}$ century skills. Nova York: Springer, p. 17-66.

Black, P., William, D. (1998). Assessment and classroom learning. Assessment in Education: Principles. Policy \& Practice, 5, (1), p. 7-74. doi: 10.1080/0969595980050102.

Branch, R. M. (2010). Instructional Design: The ADDIE Approach. New York: Springer.

Brennan, K., Resnick, M. (2012). New frameworks for studying and assessing the development of computational thinking. In: Proceedings of the Annual Meeting of the American Educational Research Association, Vancouver, Canada. [GS Search].

Chai, C. S., Deng, F., Tsai, P. Koh, J. H. L. \& Tsai, C. (2015). Assessing multidimensional students' perceptions of twenty-first-century learning practices. Asia Pacific Education Review, 16(3), 389-398. doi: 10.1007/s12564-015-9379-4. [GS Search].

Claro, M., Preiss, D. D., Martín, E. S., Jara, I., Hinostroza, E., Valenzuela, S., Cortes, F. \& Nussbaum, M. (2012). Assessment of 21 st century ICT skills in Chile: Test design and results from high school level students. Computers \& Education, 59(3), 1042-1053. doi: 10.1016/j.compedu.2012.04.004. [GS Search].

Cohen, J. (1998). Statistical Power Analysis for the Behavioral Sciences. New York: Routledge Academic.

Cronbach, L. J. (1951). Coefficient alpha and the internal structure of tests. Psychometrika, 16, p. 297-334. doi: 10.1007/BF02310555. [GS Search].

CSTA (The CSTA Standards Task Force). (2017). CSTA K-12 Computer Science Standards Revised 2017, New York: ACM.

Dede, C. (2010). Comparing frameworks for 21 St century skills. In J. Bellanca \& R. Brandt (Eds.), $21^{\text {st }}$ century skills (51-76). Bloomington: Solution Tree Press. [GS Search]. 
Delors, J., Mufti, I. A., Amagi, I., Carneiro, R., Chung, F., Geremek, B., Gorham, W., Kornhauser, A., Manley, M., Quero, M. P., Savané, M., Singh, K., Stavenhagen, R., Suhr, M. W. \& Nanzhao, Z. (1996). Learning: The treasure within. Paris: UNESCO. Disponível em: http://unesdoc.unesco.org/images/0010/001095/109590eo.pdf

Devellis, R. F. (2016). Scale Development: Theory and Applications. Beverly Hills: SAGE Publications. [GS Search].

Drasgow, F. (1986). Polychoric and polyserial correlations. In: Kotz, S., Johnson, N. L. Encyclopedia of Statistical Sciences, New York: John Wiley, 68-74.

Duckworth, A. L., Peterson, C., Matthews, M. D., Kelly, D. R. (2007). Grit: perseverance and passion for long-term goals. Journal of Personality and Social Psychology, 92(6), $1087-$ 1101. doi: 10.1037/0022-3514.92.6.1087. [GS Search].

EC (European Commission). (2017). Coding - the 21st century skill. Disponível em: https://ec.europa.eu/digital-single-market/en/coding-21st-century-skill

Griffin, P. \& Care, E. (2014). Developing learners' collaborative problem solving skills. Melbourne: Melbourne Graduate School of Education. Disponível em: http://vplearningdiaries.weebly.com/uploads/9/4/9/8/9498170/developing_learners collaborative pr oblem solving_ $p$ griffin.pdf

Grover S. \& Pea R. (2013). Computational Thinking in K-12 a Review of the State of the Field. Educational Researcher, 42(1), 38-43. doi: 10.3102\%2F0013189X12463051. [GS Search].

Hattie, J. \& Timperley, H. (2007) The power of feedback. Review of educational research, 77(1), 81-112. doi: $10.3102 \% 2 \mathrm{~F} 003465430298487$.

IFL (Institute for the Future of Learning) Assessing the Learning that Matters Most. 2015. Disponível em: https://static1.squarespace.com/static/52c8c03fe4b0da03983675b4/t/5653bcaae4b0bd925c0 $\underline{\text { 80e46/1448328362680/IFL+Report_rev_11.18.15+\%282\%29.pdf }}$

Ihantola, P., Ahoniemi, T., Karavirta, V., Seppälä, O. (2010) Review of recent systems for automatic assessment of programming assignments. In: Proceedings of the 10th Koli Calling International Conference on Computing Education Research, Koli, Finlandia. doi: 10.1145/1930464.1930480. [GS Search].

K-12 Computer Science Framework. 2016. Disponível em: http://www.k12cs.org

Kang, M., Heo, H., Jo, I., Shin, J. \& Seo, J. (2010). Developing an Educational Performance Indicator for New Millennium Learners. Journal of Research on Technology in Education, 43(2). doi: 10.1080/15391523.2010.10782567. [GS Search].

Kasunic, M. (2005). Designing an effective survey. Handbook CMU/SEI-2005-HB-004, Software Engineering Institute. Pittsburgh: Carnegie Mellon University.

Keuning, H., Jeuring, J., Heeren, B. (2016) Towards a Systematic Review of Automated Feedback Generation for Programming Exercises. In: Proceedings of the ACM Conference on Innovation and Technology in Computer Science Education, Arequipa, Peru. doi: 10.1145/2899415.2899422. [GS Search].

Kivunja, C. (2014). Do You Want Your Students to Be Job-Ready with 21st Century Skills? Change Pedagogies: A Pedagogical Paradigm Shift from Vygotskyian Social Constructivism to Critical Thinking, Problem Solving and Siemens' Digital Connectivism. International Journal of Higher Education, 3(3), 81-91. [GS Search]. 
Kyllonen, P. C. (2012). Measurement of 21st Century Skills Within the Common Core State Standards. In: Proceedings of Invitational Research Symposium on Technology Enhanced Assessments, 7-8. [GS Search].

Lau, W. W. F. \& Yuen, A. H. K. (2014). Developing and validating of a perceived ICT literacy scale for junior secondary school students: Pedagogical and educational contributions. Computers \& Education, 78, 1-9. doi: 10.1016/j.compedu.2014.04.016. [GS Search].

Losby, J., Wetmore, A. (2012). Jan Losby and Anne Wetmore on Likert Scales - Odd or Even?, Disponível em: http://aea365.org/blog/jan-losby-and-anne-wetmore-likert-scales-odd-oreven/

Masethe, M. A. Masethe, H. D. Odunaike, S. A. (2017). Scoping Review of Learning Theories in the 21th Century. In: Proceedings of the World Congress on Engineering and Computer Science, San Francisco, CA, EUA.

Melhem, A. (2002). Modelos de avaliação escolar utilizados em sala de aula - Uma análise nos cursos de administração na universidade federal do espirito santo (UFES) e de uma faculdade particular: Insumos para o aperfeiçoamento da gestão educacional. Dissertação (Mestrado em Administração), Fundação Getúlio Vargas, Rio de Janeiro, Brasil. [GS Search].

Merril, D. C., Reiser, B. J., Ranney, M., Trafton, J. G. (1992) Effective tutoring techniques: A comparison of human tutors and intelligent tutoring systems. Journal of the Learning Sciences, 2(3), 277-305. doi: 10.1207/s15327809jls0203 2. [GS Search].

Morelli R., Lanerolle, T., Lake, P., Limardo, N., Tamostu, E. \& Uche, C. (2010). Can Android App Inventor Bring Computational Thinking to K-12? Hartford, CT: Trinity College Hartford. [GS Search].

O’Neil, H. F., Schacter, J. (1997) Testing Specifications for Problem Solving Assessments. Relatório técnico, Los Angeles: University of California. 463. Disponível em: https://cresst.org/wp-content/uploads/TECH463.pdf

OECD (Organization for Economic Co-operation and Development), Collaborative problemsolving framework. Paris: OECD Publishing, 2015.

Partnership for $21^{\text {st }}$ Century Skills (P21). (2015). P21 framework definitions. Disponível em: http://www.p21.org/storage/documents/P21_Framework_Definitions.pdf

Partnership for 21st Century Skills (P21). (2017). Computer Science: A playground for $21^{\text {st }}$ century skills. Disponível em: http://www.p21.org/news-events/p21blog/2128-computerscience-a-playground-for-21st-century-skills

Petway, K. T., Rikoon, S. H., Brenneman, M. W., Burrus, J., Roberts, R. D. (2016). Development of the Mission Skills Assessment and Evidence of Its Reliability and Internal Structure. Relatório de pesquisa, ETS (Educational testing service). doi: 10.1002/ets2.12107. Disponível em: https://www.ets.org/Media/Research/pdf/session5kyllonen-paper-tea2012.pdf. [GS Search].

Rosen, Y. \& Tager, M. (2014). Computer-based Performance Assessment of Creativity Skills: A Pilot Study. In: Proceedings of International Association for Educational Assessment Conference, Singapore. [GS Search].

Rosen, Y. (2015). Computer-based Assessment of Collaborative Problem Solving: Exploring the Feasibility of Human-to-Agent Approach. International Journal of Artificial Intelligence in Education, 25(3), 380-406. doi: 10.1007/s40593-015-0042-3. [GS Search]. 
Ross, J. A. (2006) The reliability, validity, and utility of self-assessment. Practical Assessment, Research \& Evaluation, 11(10), 1-13. [GS Search].

Rusman, E., Boon, J., Martínez-Monés, A., Rodríguez-Triana, M. J., Simeos, R. (2003) Towards the Use of New Methods for Formative e-Assessments of 21st Century Skills in Schools. In: Proc. of Technology Enhanced Formative Assessment Workshop at EC-TEL. Paphos, Chipre. [GS Search].

Sadler, D. R. (1989) Formative assessment and the design of instructional systems. Instructional Science, 18(2), 119-144. doi: 10.1007/BF00117714. [GS Search].

Shute, V. J. (2008) Focus on formative feedback. Review of Educational Research, 78(1), 153189. doi: $10.3102 \% 2 \mathrm{~F} 0034654307313795$. [GS Search].

Siddiq, F., Gochyyev, P. \& Wilson, M. (2017). Learning in Digital Networks - ICT Literacy: A novel assessment of students' 21st century skills. Computer \& Education, 109, 11-37. doi: 10.1016/i.compedu.2017.01.014. [GS Search].

Sitzman, T., Ely, K., Brown, K. G., Bauer, K. N. (2010) Self-assessment of knowledge: A cognitive learning or affective measure? Academy of Management Learning \& Education, 9(2), 169-191. doi: 10.5465/amle.9.2.zqr169. [GS Search].

Sociedade Brasileira de Computação (SCB). (2017). Referenciais de Formação em Computação: Educação Básica. Disponível http://www.sbc.org.br/files/ComputacaoEducacaoBasica-versaofinal-julho2017.pdf

Soland, J., Hamilton, L. S. \& Stecher, B. M. (2013) Measuring 21st Century Competencies, Guidance for Educators. Global Cities Education Network Report.

Stegeman, M., Barendsen, E., Smeters, S. (2016) Designing a rubric for feedback on code quality in programming courses. In: Proceedings of the 16th Koli Calling International Conference on Computing Education Research, Koli: Finlandia. doi: 10.1145/2999541.2999555. [GS Search].

Susnea, I. \& Vasiliu, G. (2016). A Fuzzy Logic Software Tool and a New Scale for the Assessment of Creativity. International Journal of Computers Communications \& Control, 11(3), 441-449. doi: 10.15837/ijccc.2016.3.2192. [GS Search].

Topping, K. (2003) Self and Peer Assessment in School and University: Reliability, Validity and Utility. In M. Segers, F. Dochy, \& E. Cascallar (Eds.), Optimising New Modes of Assessment: In Search of Qualities and Standards, Dordrecht: Kluwer Academic Publishers. [GS Search].

Torrance, H. (1995) Evaluating authentic assessment: Problems and possibilities in new approaches to assessment. Buckingham: Open University Press.

Trochim, W. M. \& Donnelly, J. P. (2008). Research Methods Knowledge Base. Mason: Atomic Dog Publishing.

Tsai, M., Wang, C., Hsu, P. (2018). Developing the Computer Programming Self-Efficacy Scale for Computer Literacy Education. Journal of Educational Computing Research, 1-16. doi: 10.1177\%2F0735633117746747. [GS Search].

Voogt, J., Roblin, N. P. (2012). A comparative analysis of international frameworks for $21^{\text {st }}$ century competences: Implications for national curriculum policies. Journal of Curriculum Studies, 44(3), 299-321. doi: 10.1080/00220272.2012.668938. [GS Search]. 
Wangenheim, C. G., Alves, N. C., Weber, A. R. (2017). Resumo do K-12 Computer Science Standards (Versão 2017). Relatório Técnico do INCoD/INE/UFSC, Florianópolis/SC.

Ward, J. D., Lee, C. L. (2002) A review of problem-based learning. Journal of Family and Consumer Sciences Education, 20(1), 16-26.

Wiggins, G. P. (1993) Assessing student performance: Exploring the purpose and limits of testing. San Francisco: Jossey-Bass. 